\title{
SPARSE SPECTRAL APPROXIMATIONS OF HIGH-DIMENSIONAL PROBLEMS BASED ON HYPERBOLIC CROSS*
}

\author{
JIE SHEN $^{\dagger}$ AND LI-LIAN WANG ${ }^{\ddagger}$
}

\begin{abstract}
Hyperbolic cross approximations by some classical orthogonal polynomials/functions in both bounded and unbounded domains are considered in this paper. Optimal error estimates in proper anisotropic weighted Korobov spaces for both regular hyperbolic cross approximations and optimized hyperbolic cross approximations are established. These fundamental approximation results indicate that spectral methods based on hyperbolic cross approximations can be effective for treating certain high-dimensional problems and will serve as basic tools for analyzing sparse spectral methods in high dimensions.
\end{abstract}

Key words. hyperbolic cross, high-dimensional problems, sparse spectral methods, orthogonal polynomials, convergence rate

AMS subject classifications. $65 \mathrm{~N} 35,65 \mathrm{~N} 22,65 \mathrm{~F} 05$

DOI. $10.1137 / 090765547$

1. Introduction. Many scientific and engineering applications require solving high-dimensional partial differential equations (PDEs). Examples include fundamental equations in physics such as Boltzmann equations, Fokker-Planck equations, Schrödinger equations, and mathematical models in finance (cf., for instance, [2] and the references therein).

Current spectral methods for solving PDEs in multidimensions are usually based on tensor product formulation. Assuming $N$ points are used in each direction, the total number of unknowns for the spatial variables is $M=N^{d}$, where $d$ is the dimension. Thus, even for problems with a moderate dimension, current computing power does not allow a reasonable full tensor product grid needed to resolve the physical solution. Another way to look at the difficulty is through the error decay rate in terms of total number of unknowns. Let $X_{N}$ be an approximation space based on the full tensor product grid with $N$ points in each direction. A typical error estimate is of the form

$$
\inf _{v_{N} \in X_{N}}\left\|u-v_{N}\right\|_{L^{2}} \lesssim N^{-r}\|u\|_{H^{r}} \lesssim M^{-\frac{r}{d}}\|u\|_{H^{r}}
$$

where $H^{r}$ is the usual Sobolev space, and the notation " $\lesssim$ " means " $\leq$ " up to a positive constant independent of $N$ and $u$. Hence, the convergence rate deteriorates rapidly as $d$ increases, leading to the so-called curse of dimensionality (cf. [5]). Another viewpoint for the curse of dimensionality related to the full tensor grid is that it samples only $M^{\frac{1}{d}}$ points in each coordinate, explaining why the approximation error usually decays as powers of $M^{-\frac{1}{d}}$.

*Received by the editors July 20, 2009; accepted for publication (in revised form) May 9, 2010; published electronically July 22, 2010.

http://www.siam.org/journals/sinum/48-3/76554.html

†School of Mathematical Sciences, Xiamen University, China, and Department of Mathematics, Purdue University, West Lafayette, IN (shen7@purdue.edu). The work of this author was partially supported by AFOSR grant FA9550-08-1-0416 and NFS grant DMS-0915066.

${ }^{\ddagger}$ Division of Mathematical Sciences, School of Physical and Mathematical Sciences, Nanyang Technological University, Singapore (lilian@ntu.edu.sg). The work of this author was partially supported by Singapore AcRF Tier 1 grant RG58/08, Singapore MOE grant T207B2202, and Singapore grant NRF2007IDM-IDM002-010.

1087 
In order to understand better the crucial issues in high-dimensional approximation, we consider, for example, the $d$-dimensional Poisson-type equation

$$
\alpha u-\Delta u=f, \quad x \in \Omega=(-1,1)^{d} ;\left.\quad u\right|_{\partial \Omega}=0,
$$

which has to be solved repeatedly in many high-dimensional applications at every time step and/or at every iteration of an iterative procedure.

Let $X_{N}$ be a finite-dimensional space and $I_{N}$ an interpolation operator, both to be specified. A weighted spectral Galerkin method for (1.2) is to find $u_{N} \in X_{N}$ such that

$$
\alpha\left(u_{N}, v_{N}\right)_{\omega}-\left(\Delta u_{N}, v_{N}\right)_{\omega}=\left(I_{N} f, v_{N}\right)_{\omega} \quad \forall v_{N} \in X_{N}
$$

where $\omega$ is a positive weight function, and $(\cdot, \cdot)_{\omega}$ represents the weighted $L^{2}$ inner product. Then, in general, we have an error estimate of the form

$$
\begin{aligned}
\left\|\nabla\left(u-u_{N}\right)\right\|_{L_{\omega}^{2}} & \lesssim \inf _{v_{N} \in X_{N}}\left\|\nabla\left(u-v_{N}\right)\right\|_{L_{\omega}^{2}}+\sup _{\phi \in X_{N} ; \nabla \phi \neq 0} \frac{(f, \phi)_{\omega}-\left(I_{N} f, \phi\right)_{\omega}}{\|\nabla \phi\|_{L_{\omega}^{2}}} \\
& \lesssim \inf _{v_{N} \in X_{N}}\left\|\nabla\left(u-v_{N}\right)\right\|_{L_{\omega}^{2}}+\left\|f-I_{N} f\right\|_{L_{\omega}^{2}} .
\end{aligned}
$$

The first term on the right-hand side is the best approximation error in $X_{N}$, while the second term represents the integration/interpolation error. The main question is how to choose $X_{N}$ and $I_{N}$ such that (1.4) has a fast convergence rate with respect to the number of degrees of freedom in $X_{N}$ and that (1.3) can be solved efficiently.

Let us first consider the choice of $X_{N}$. It is well known (cf., for instance, [20, 21]) that for multivariate periodic problems, the so-called hyperbolic cross space

$$
X_{N}=\operatorname{span}\left\{\exp (\mathrm{i} \boldsymbol{k} \cdot \boldsymbol{x}):|\boldsymbol{k}|_{\text {mix }}:=\prod_{j=1}^{d} \max \left\{\left|k_{j}\right|, 1\right\} \leq N\right\}
$$

provides optimal approximation properties for functions with periodic bounded mixed derivatives, or more precisely, in the Korobov space

$$
\mathcal{K}_{p}^{m}\left((0,2 \pi)^{d}\right):=\left\{u=\sum_{\boldsymbol{k} \in \mathbb{Z}^{d}} c_{\boldsymbol{k}} \exp (\mathrm{i} \boldsymbol{k} \cdot \boldsymbol{x}):\|u\|_{\mathcal{K}_{p}^{m}}^{2}:=\sum_{\boldsymbol{k} \in \mathbb{Z}^{d}}|\boldsymbol{k}|_{\text {mix }}^{2 m}\left|c_{\boldsymbol{k}}\right|^{2}<\infty\right\} .
$$

In fact, one can easily establish the following result (cf., for instance, [15]).

TheOREM 1.1. For $u \in \mathcal{K}_{p}^{m}\left((0,2 \pi)^{d}\right)$ and $0 \leq l \leq m$, we have

$$
\inf _{u_{N} \in X_{N}}\left\|u-u_{N}\right\|_{\mathcal{K}_{p}^{l}} \leq C N^{l-m}\|u\|_{\mathcal{K}_{p}^{m}}
$$

where the positive constant $C$ depends on $d$ and $m$, but is independent of $N$ and $u$.

Since the cardinality of $X_{N}$ is of order $M=N(\log N)^{d-1}$ (cf., e.g., [15]), the above result indicates that the hyperbolic cross approximation error deteriorates very mildly as $d$ increases. Therefore, it is natural, for nonperiodic problems, to choose $X_{N}$ to be the hyperbolic cross spaces associated with orthogonal polynomials/functions, such as Jacobi polynomials for bounded domains and Hermite and Laguerre functions for unbounded domains.

Now let us consider the choice of the interpolation operator $I_{N}$. Once the approximation space $X_{N}$ is fixed, one needs to choose a set of nodes $\Sigma_{N}$ such that the interpolation operator $I_{N}: C(\bar{\Omega}) \rightarrow X_{N}$ with $\left(I_{N} f\right)(\boldsymbol{x})=f(\boldsymbol{x})$ for all $\boldsymbol{x} \in \Sigma_{N}$ 
is well defined, and more importantly, the transforms between the expansion coefficients in terms of the basis of $X_{N}$ and the function values at $\Sigma_{N}$ can be performed by a fast and stable algorithm. It has been observed (cf. $[4,19])$ that for multivariate periodic functions, one can define such an interpolation operator onto $X_{N}$ (defined in (1.5)) based on the sparse grids constructed by Smolyak's algorithm, and the forward and backward transforms can be performed by using the fast Fourier transform (FFT). Since the Fourier expansion (in $\theta$ ) and the Chebyshev expansion (in $x$ ) are linked by the relation $x=\cos \theta$, it is natural, for nonperiodic problems, to define the interpolation operator based on the sparse grids $\Sigma_{N}$ constructed by Smolyak's algorithm based on the nested Chebyshev-Gauss-Lobatto quadrature. Thanks to the fact that the Chebyshev polynomials (resp., Chebyshev-Gauss-Lobatto points) are the image of trigonometric polynomials (resp., equally spaced points) under the mapping $\theta=\cos ^{-1} x$, the transforms between the expansion coefficients in the Chebyshev hyperbolic cross $X_{N}$ and the function values at the Chebyshev sparse grid $\Sigma_{N}$ can also be performed in a fast and stable manner using the FFT.

Approximations by hyperbolic cross and sparse grid have received much attention in recent years (cf. $[29,30,7,14,2]$ and the references therein). While most of the studies have concentrated on the $h$-type wavelet or finite element approximations (cf., for instance, $[12,22,23,10]$ or the $p$-type Fourier approximations (cf., for instance, $[15,13])$, only a few papers have been devoted to the polynomial approximations using sparse grids generated from the Chebyshev-Gauss-Lobatto points (cf., for instance, $[3,27])$. To the best of our knowledge, there is no systematical error analysis for hyperbolic cross approximations based on classical polynomials/functions. The main purpose of this paper is to establish optimal error estimates for the hyperbolic cross approximations with respect to some classical orthogonal polynomials/functions in anisotropic weighted Korobov spaces. In particular, we shall prove the following result for the Jacobi hyperbolic cross approximation.

THEOREM 1.2.

$$
\inf _{u_{N} \in X_{N}}\left\|u-u_{N}\right\|_{\mathcal{K}_{\boldsymbol{\alpha}, \boldsymbol{\beta}}^{l}\left(I^{d}\right)} \leq C N^{l-m}|u|_{\mathcal{K}_{\boldsymbol{\alpha}, \boldsymbol{\beta}}^{m}\left(I^{d}\right)}, \quad 0 \leq l \leq m,
$$

where the positive constant $C$ depends on $d$ and $m$, but is independent of $N$ and $u$.

In the above, $X_{N}:=X_{N}^{\alpha, \beta}$ (cf. (2.33) and (2.34) below) is the hyperbolic cross approximation space associated with the Jacobi polynomials, and the space $\mathcal{K}_{\boldsymbol{\alpha}, \boldsymbol{\beta}}^{l}\left(I^{d}\right)$ is the anisotropic Jacobi weighted Korobov space defined in (2.37) and (2.38) below. Note that the above result is analogous to Theorem 1.1 but here the proper functional spaces are the anisotropic Jacobi weighted Korobov spaces, instead of the spaces with bounded mixed derivatives in Theorem 1.1. The use of anisotropic weights allows us to obtain better convergence rates for functions with corner and boundary singularities (see, e.g., [10]). The proof of Theorem 1.2 is given in section 2 (see Corollary 2.1), where an explicit estimate of the constant $C$ is given. We shall also establish similar approximation results for hyperbolic cross approximations by Hermite and Laguerre functions.

The rest of the paper is organized as follows. In section 2, we consider both the full tensor grid and hyperbolic cross approximations based on Jacobi polynomials in Jacobi-weighted Korobov spaces. In section 3, we establish similar results for the hyperbolic cross approximations by Hermite and Laguerre functions in unbounded domains. As an example of applications, we construct the sparse spectral Galerkin method for Poisson-type equations in the hypercube in section 4, and we finish the paper with some concluding remarks in the last section. 
2. Hyperbolic cross approximation in Jacobi-weighted Korobov-type spaces. In this section, we study the Jacobi approximations in Jacobi-weighted Sobolev spaces and Korobov-type spaces using full grids and sparse grids.

2.1. Notation. We first introduce some notation to be used throughout the paper.

- Denote by $\mathbb{R}$ (resp., $\mathbb{N}$ ) the set of all real numbers (resp., positive integers), and let $\mathbb{N}_{0}=\mathbb{N} \cup\{0\}$.

- For $d \in \mathbb{N}$, we use boldface lowercase letters to denote $d$-dimensional multiindexes and vectors, e.g., $\boldsymbol{k}=\left(k_{1}, \ldots, k_{d}\right) \in \mathbb{N}_{0}^{d}$ and $\boldsymbol{\alpha}=\left(\alpha_{1}, \ldots, \alpha_{d}\right) \in \mathbb{R}^{d}$. Also, let $\mathbf{1}=(1,1, \ldots, 1) \in \mathbb{N}^{d}$, and let $\boldsymbol{e}_{i}=(0, \ldots, 1, \ldots, 0)$ be the $i$ th unit vector in $\mathbb{R}^{d}$. For a scalar $s \in \mathbb{R}$, we define the following componentwise operations:

$$
\boldsymbol{\alpha}+\boldsymbol{k}=\left(\alpha_{1}+k_{1}, \ldots, \alpha_{d}+k_{d}\right), \quad \boldsymbol{\alpha}+s:=\boldsymbol{\alpha}+s \mathbf{1}=\left(\alpha_{1}+s, \ldots, \alpha_{d}+s\right)
$$

and we use the following conventions:

$$
\boldsymbol{\alpha} \geq \boldsymbol{k} \Leftrightarrow \forall_{1 \leq j \leq d} \alpha_{j} \geq k_{j} ; \boldsymbol{\alpha} \geq s \Leftrightarrow \boldsymbol{\alpha} \geq s \mathbf{1} \Leftrightarrow \forall_{1 \leq j \leq d} \alpha_{j} \geq s .
$$

- We denote

$$
|\boldsymbol{k}|_{1}=\sum_{j=1}^{d} k_{j}, \quad|\boldsymbol{k}|_{\infty}=\max _{1 \leq j \leq d} k_{j}, \quad \bar{k}_{j}=\max _{1 \leq j \leq d}\left\{1, k_{j}\right\}, \quad|\boldsymbol{k}|_{\text {mix }}=\prod_{j=1}^{d} \bar{k}_{j} .
$$

- Let $\Lambda:=(a, b) \subset \mathbb{R}$ be a generic finite or infinite interval, and denote $\Lambda^{d}:=$ $(a, b)^{d}$. Given a multivariate function $u(\boldsymbol{x})$, we denote the $|\boldsymbol{k}|_{1}$ th (mixed) partial derivative by

$$
\boldsymbol{\partial}_{\boldsymbol{x}}^{\boldsymbol{k}} u=\frac{\partial^{|\boldsymbol{k}|_{1}} u}{\partial x_{1}^{k_{1}} \cdots \partial x_{d}^{k_{d}}}=\partial_{x_{1}}^{k_{1}} \cdots \partial_{x_{d}}^{k_{d}} u
$$

In particular, we denote $\boldsymbol{\partial}_{\boldsymbol{x}}^{s} u:=\boldsymbol{\partial}_{\boldsymbol{x}}^{s \mathbf{1}} u=\boldsymbol{\partial}_{\boldsymbol{x}}^{(s, s, \ldots, s)} u$.

Given a generic weight function $\boldsymbol{\omega}(\boldsymbol{x})$ in $\Lambda^{d}$, we define the weighted Sobolev spaces $H_{\boldsymbol{\omega}}^{r}\left(\Lambda^{d}\right)$ with the norm $\|\cdot\|_{r, \boldsymbol{\omega}, \Lambda^{d}}$ as in [1]. In particular, we have $L_{\boldsymbol{\omega}}^{2}\left(\Lambda^{d}\right)=H_{\boldsymbol{\omega}}^{0}\left(\Lambda^{d}\right)$ and denote its inner product and norm by $(\cdot, \cdot)_{\boldsymbol{\omega}, \Lambda^{d}}$ and $\|\cdot\|_{\boldsymbol{\omega}, \Lambda^{d}}$, respectively. If $\boldsymbol{\omega}(\boldsymbol{x}) \equiv 1$, we drop $\boldsymbol{\omega}$ in the above notation.

- For any $N \in \mathbb{N}, \mathcal{P}_{N}(\Lambda)$ denotes the set of all real polynomials of degree $\leq N$ in $\Lambda$.

- The notation $A \simeq B$ means that the ratio $A / B$ with $B \neq 0$ tends to 1 in certain limiting process.

2.2. Jacobi polynomials. Now, we recall some relevant properties of the Jacobi polynomials (cf. [28]). Let $\omega^{\alpha, \beta}(x)=(1-x)^{\alpha}(1+x)^{\beta}$ be the Jacobi weight function defined in $I:=(-1,1)$. The one-dimensional Jacobi polynomials, denoted by $J_{n}^{\alpha, \beta}(x)(\alpha, \beta>-1)$, are the eigenfunctions of the Sturm-Liouville problem,

$$
-\frac{1}{\omega^{\alpha, \beta}(x)} \partial_{x}\left(\omega^{\alpha+1, \beta+1}(x) \partial_{x} J_{n}^{\alpha, \beta}(x)\right)=\lambda_{n}^{\alpha, \beta} J_{n}^{\alpha, \beta}(x), \quad x \in I, n \geq 0,
$$

with the corresponding eigenvalues

$$
\lambda_{n}^{\alpha, \beta}=n(n+\alpha+\beta+1) .
$$

Copyright (c) by SIAM. Unauthorized reproduction of this article is prohibited. 
For $\alpha, \beta>-1$, the family of Jacobi polynomials forms a complete orthogonal system in $L_{\omega^{\alpha, \beta}}^{2}(I)$, and hereafter we assume that they are normalized so that

$$
\int_{-1}^{1} J_{n}^{\alpha, \beta}(x) J_{n^{\prime}}^{\alpha, \beta}(x) \omega^{\alpha, \beta}(x) d x=\delta_{n n^{\prime}},
$$

where $\delta_{n n^{\prime}}$ is the Kronecker delta.

An important property of the normalized Jacobi polynomials is

$$
\partial_{x} J_{n}^{\alpha, \beta}(x)=\sqrt{\lambda_{n}^{\alpha, \beta}} J_{n-1}^{\alpha+1, \beta+1}(x), \quad n \geq 1 .
$$

Applying this formula recursively leads to

$$
\partial_{x}^{k} J_{n}^{\alpha, \beta}(x)=\sqrt{\chi_{n, k}^{\alpha, \beta}} J_{n-k}^{\alpha+k, \beta+k}(x), \quad n \geq k \geq 1,
$$

where the factor

$$
\chi_{n, k}^{\alpha, \beta}=\prod_{j=0}^{k-1} \lambda_{n-j}^{\alpha+j, \beta+j}=\frac{n ! \Gamma(n+k+\alpha+\beta+1)}{(n-k) ! \Gamma(n+\alpha+\beta+1)}, \quad n \geq k \geq 1 .
$$

One verifies readily that for all $n \geq j+1 \geq 1$ and $\alpha, \beta>-1$,

$$
\lambda_{n-j-1}^{\alpha+j+1, \beta+j+1}-\lambda_{n-j}^{\alpha+j, \beta+j}=-(2 j+\alpha+\beta+2)<0 .
$$

Hence, $\lambda_{n-j}^{\alpha+j, \beta+j}$ is strictly descending with respect to $j$, and there holds

$$
\left(\lambda_{n-k+1}^{\alpha+k-1, \beta+k-1}\right)^{k} \leq \chi_{n, k}^{\alpha, \beta} \leq\left(\lambda_{n}^{\alpha, \beta}\right)^{k}, \quad n \geq k \geq 1, \quad \alpha, \beta>-1 .
$$

For notational convenience, we extend the definition of $\chi_{n, k}^{\alpha, \beta}$ to all $n, k \geq 0$ by defining

$$
\chi_{n, k}^{\alpha, \beta}= \begin{cases}1 & \text { if } k=0, n \geq 0, \\ 0 & \text { if } k>n \geq 0 .\end{cases}
$$

We deduce from (2.7), (2.9), and (2.13) that $\left\{\partial_{x}^{k} J_{n}^{\alpha, \beta}\right\}_{n=k}^{\infty}$ are mutually orthogonal with respect to the weight function $\omega^{\alpha+k, \beta+k}$, and

$$
\left\|\partial_{x}^{k} J_{n}^{\alpha, \beta}\right\|_{\omega^{\alpha+k, \beta+k, I}}^{2}=\chi_{n, k}^{\alpha, \beta} \quad \forall n, k \geq 0 \text { and } \forall \alpha, \beta>-1 .
$$

Defining the $d$-dimensional tensorial Jacobi polynomial and Jacobi weight function as

$$
\boldsymbol{J}_{\boldsymbol{n}}^{\boldsymbol{\alpha}, \boldsymbol{\beta}}(\boldsymbol{x})=\prod_{j=1}^{d} J_{n_{j}}^{\alpha_{j}, \beta_{j}}\left(x_{j}\right), \quad \boldsymbol{\omega}^{\boldsymbol{\alpha}, \boldsymbol{\beta}}(\boldsymbol{x})=\prod_{j=1}^{d} \omega^{\alpha_{j}, \beta_{j}}\left(x_{j}\right) \forall \boldsymbol{\alpha}, \boldsymbol{\beta}>-1, \boldsymbol{x} \in I^{d},
$$

we derive from (2.9) and (2.14) that

$$
\boldsymbol{\partial}_{\boldsymbol{x}}^{\boldsymbol{k}} \boldsymbol{J}_{\boldsymbol{n}}^{\boldsymbol{\alpha}, \boldsymbol{\beta}}(\boldsymbol{x})=\sqrt{\chi_{\boldsymbol{n}, \boldsymbol{k}}^{\boldsymbol{\alpha}, \boldsymbol{\beta}}} \boldsymbol{J}_{\boldsymbol{n}-\boldsymbol{k}}^{\boldsymbol{\alpha}+\boldsymbol{k}, \boldsymbol{\beta}+\boldsymbol{k}}(\boldsymbol{x}) \quad \text { with } \quad \chi_{\boldsymbol{n}, \boldsymbol{k}}^{\boldsymbol{\alpha}, \boldsymbol{\beta}}=\prod_{j=1}^{d} \chi_{n_{j}, k_{j}}^{\alpha_{j}, \beta_{j}}
$$

Copyright (C) by SIAM. Unauthorized reproduction of this article is prohibited. 
and

$$
\int_{I^{d}} \partial_{x}^{k} J_{n}^{\alpha, \beta}(x) \partial_{x}^{k} J_{m}^{\alpha, \beta}(x) \omega^{\alpha+k, \beta+k}(x) d x=\chi_{n, k}^{\alpha, \beta} \delta_{n m},
$$

where $\boldsymbol{n}, \boldsymbol{k} \geq 0, \boldsymbol{\alpha}, \boldsymbol{\beta}>-1$, and $\boldsymbol{\delta}_{\boldsymbol{n} \boldsymbol{m}}=\prod_{j=1}^{d} \delta_{n_{j} m_{j}}$.

For any $u \in L_{\boldsymbol{\omega}^{\alpha, \boldsymbol{\beta}}}^{2}\left(I^{d}\right)$, we write

$$
u(\boldsymbol{x})=\sum_{\boldsymbol{n} \geq 0} \hat{u}_{\boldsymbol{n}}^{\boldsymbol{\alpha}, \boldsymbol{\beta}} \boldsymbol{J}_{\boldsymbol{n}}^{\boldsymbol{\alpha}, \boldsymbol{\beta}}(\boldsymbol{x}) \quad \text { with } \quad \hat{u}_{\boldsymbol{n}}^{\boldsymbol{\alpha}, \boldsymbol{\beta}}=\int_{I^{d}} u(\boldsymbol{x}) \boldsymbol{J}_{\boldsymbol{n}}^{\boldsymbol{\alpha}, \boldsymbol{\beta}}(\boldsymbol{x}) \boldsymbol{\omega}^{\boldsymbol{\alpha}, \boldsymbol{\beta}}(\boldsymbol{x}) d \boldsymbol{x} .
$$

Formally, we have $\boldsymbol{\partial}_{\boldsymbol{x}}^{\boldsymbol{k}} u=\sum_{\boldsymbol{n} \geq \boldsymbol{k}} \hat{u}_{\boldsymbol{n}}^{\boldsymbol{\alpha}, \boldsymbol{\beta}} \boldsymbol{\partial}_{\boldsymbol{x}}^{\boldsymbol{k}} \boldsymbol{J}_{\boldsymbol{n}}^{\boldsymbol{\alpha}, \boldsymbol{\beta}}$, and by the orthogonality (2.17),

$$
\left\|\boldsymbol{\partial}_{\boldsymbol{x}}^{\boldsymbol{k}} u\right\|_{\boldsymbol{\omega}^{\alpha+\boldsymbol{k}, \boldsymbol{\beta}+\boldsymbol{k}, I^{d}}}^{2}=\sum_{\boldsymbol{n} \geq \boldsymbol{k}} \chi_{\boldsymbol{n}, \boldsymbol{k}}^{\boldsymbol{\alpha}, \boldsymbol{\beta}}\left|\hat{u}_{\boldsymbol{n}}^{\boldsymbol{\alpha}, \boldsymbol{\beta}}\right|^{2} \stackrel{(2.13)}{=} \sum_{\boldsymbol{n} \in \mathbb{N}_{0}^{d}} \chi_{\boldsymbol{n}, \boldsymbol{k}}^{\boldsymbol{\alpha}, \boldsymbol{\beta}}\left|\hat{u}_{\boldsymbol{n}}^{\boldsymbol{\alpha}, \boldsymbol{\beta}}\right|^{2} .
$$

In what follows, we consider the approximation of functions in $L_{\boldsymbol{\omega}^{\alpha, \beta}}^{2}\left(I^{d}\right)$ by multivariate Jacobi expansions.

2.3. Multivariate Jacobi approximations. Let $\Upsilon_{N} \subset \mathbb{N}_{0}^{d}$ be an index set to be specified later, and define the finite-dimensional polynomial space:

$$
X_{N}^{\boldsymbol{\alpha}, \boldsymbol{\beta}}:=\operatorname{span}\left\{\boldsymbol{J}_{\boldsymbol{n}}^{\boldsymbol{\alpha}, \boldsymbol{\beta}}: \boldsymbol{n} \in \Upsilon_{N}\right\} .
$$

The orthogonal projection $\boldsymbol{\pi}_{N}^{\boldsymbol{\alpha}, \boldsymbol{\beta}}: L_{\boldsymbol{\omega}^{\boldsymbol{\alpha}, \boldsymbol{\beta}}}^{2}\left(I^{d}\right) \rightarrow X_{N}^{\boldsymbol{\alpha}, \boldsymbol{\beta}}$ is defined by

$$
\int_{I^{d}}\left(\boldsymbol{\pi}_{N}^{\boldsymbol{\alpha}, \boldsymbol{\beta}} u-u\right) v_{N} \boldsymbol{\omega}^{\boldsymbol{\alpha}, \boldsymbol{\beta}} d \boldsymbol{x}=0 \quad \forall v_{N} \in X_{N}^{\boldsymbol{\alpha}, \boldsymbol{\beta}}
$$

or, equivalently,

$$
\left(\boldsymbol{\pi}_{N}^{\boldsymbol{\alpha}, \boldsymbol{\beta}} u\right)(\boldsymbol{x})=\sum_{\boldsymbol{n} \in \Upsilon_{N}} \hat{u}_{\boldsymbol{n}}^{\boldsymbol{\alpha}, \boldsymbol{\beta}} \boldsymbol{J}_{\boldsymbol{n}}^{\boldsymbol{\alpha}, \boldsymbol{\beta}}(\boldsymbol{x}) .
$$

Now, we analyze the error " $\boldsymbol{\pi}_{N}^{\boldsymbol{\alpha}, \boldsymbol{\beta}} u-u$ " for several typical choices of $\Upsilon_{N}$, including the usual full tensor grid and the hyperbolic cross/sparse grid.

2.3.1. Jacobi approximations on the full grid. We first consider the $d$ dimensional full tensor grid with the index set $\Upsilon_{N}:=\Upsilon_{N}^{F}=\left\{\boldsymbol{n} \in \mathbb{N}_{0}^{d}:|\boldsymbol{n}|_{\infty} \leq N\right\}$. In this case,

$$
X_{N}^{\boldsymbol{\alpha}, \boldsymbol{\beta}}=\mathcal{P}_{N}^{d} \quad \Rightarrow \quad M:=\operatorname{dim}\left(X_{N}^{\boldsymbol{\alpha}, \boldsymbol{\beta}}\right)=(N+1)^{d} .
$$

We define the $d$-dimensional Jacobi-weighted Sobolev space as an extension of the one-dimensional setting in [18]:

$$
\mathcal{B}_{\boldsymbol{\alpha}, \boldsymbol{\beta}}^{m}\left(I^{d}\right):=\left\{u: \boldsymbol{\partial}_{\boldsymbol{x}}^{\boldsymbol{k}} u \in L_{\boldsymbol{\omega}^{\boldsymbol{\alpha}+\boldsymbol{k}, \boldsymbol{\beta}+\boldsymbol{k}}}^{2}\left(I^{d}\right), \quad 0 \leq|\boldsymbol{k}|_{1} \leq m\right\} \quad \forall m \in \mathbb{N}_{0},
$$

equipped with the norm and seminorm

$$
\begin{aligned}
& \|u\|_{\mathcal{B}_{\boldsymbol{\alpha}, \boldsymbol{\beta}}^{m}\left(I^{d}\right)}=\left(\sum_{0 \leq|\boldsymbol{k}|_{1} \leq m}\left\|\boldsymbol{\partial}_{\boldsymbol{x}}^{k} u\right\|_{\boldsymbol{\omega}^{\boldsymbol{\alpha}+\boldsymbol{k}, \boldsymbol{\beta}+\boldsymbol{k}, I^{d}}}^{2}\right)^{\frac{1}{2}}, \\
& |u|_{\mathcal{B}_{\boldsymbol{\alpha}, \boldsymbol{\beta}}^{m}\left(I^{d}\right)}=\left(\sum_{j=1}^{d}\left\|\partial_{x_{j}}^{m} u\right\|_{\boldsymbol{\omega}^{\boldsymbol{\alpha}+m \boldsymbol{e}_{j}, \boldsymbol{\beta}+m \boldsymbol{e}_{j}, I^{d}}}^{2}\right)^{\frac{1}{2}},
\end{aligned}
$$

Copyright (c) by SIAM. Unauthorized reproduction of this article is prohibited. 
where $\boldsymbol{e}_{j}$ is the $j$ th unit vector of $\mathbb{R}^{d}$. It is clear that $\mathcal{B}_{\boldsymbol{\alpha}, \boldsymbol{\beta}}^{m}\left(I^{d}\right) \subseteq H_{\boldsymbol{\omega}^{\boldsymbol{\alpha}, \boldsymbol{\beta}}}^{m}\left(I^{d}\right)$ and $\mathcal{B}_{\boldsymbol{\alpha}, \boldsymbol{\beta}}^{0}\left(I^{d}\right)=L_{\boldsymbol{\omega}^{\alpha, \boldsymbol{\beta}}}^{2}\left(I^{d}\right)$.

Theorem 2.1. Given $u \in \mathcal{B}_{\boldsymbol{\alpha}, \boldsymbol{\beta}}^{m}\left(I^{d}\right)$, we have

$$
\left|\boldsymbol{\pi}_{N}^{\boldsymbol{\alpha}, \boldsymbol{\beta}} u-u\right|_{\mathcal{B}_{\boldsymbol{\alpha}, \boldsymbol{\beta}}^{l}\left(I^{d}\right)} \leq c N^{l-m}|u|_{\mathcal{B}_{\boldsymbol{\alpha}, \boldsymbol{\beta}}^{m}\left(I^{d}\right)}, \quad 0 \leq l \leq m,
$$

where $c \simeq \sqrt{2}$ for $N \gg 1$.

Proof. To simplify the presentation, we assume that $\boldsymbol{\alpha}$ and $\boldsymbol{\beta}$ are constant vectors, and $N \geq m$, since we are only interested in $N \gg 1$.

By (2.17)-(2.19) and (2.22), we have that for any $1 \leq j \leq d$,

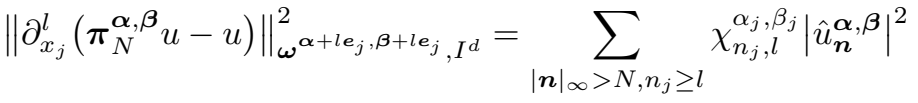

$$
\begin{aligned}
& =\sum_{\boldsymbol{n} \in \Lambda_{N}^{1, j}} \chi_{n_{j}, l}^{\alpha_{j}, \beta_{j}}\left|\hat{u}_{\boldsymbol{n}}^{\boldsymbol{\alpha}, \boldsymbol{\beta}}\right|^{2}+\sum_{\boldsymbol{n} \in \Lambda_{N}^{2, j}} \chi_{n_{j}, l}^{\alpha_{j}, \beta_{j}}\left|\hat{u}_{\boldsymbol{n}}^{\boldsymbol{\alpha}, \boldsymbol{\beta}}\right|^{2}, \quad 1 \leq j \leq d,
\end{aligned}
$$

where the index sets are

$\Lambda_{N}^{1, j}:=\left\{\boldsymbol{n} \in \mathbb{N}_{0}^{d}:|\boldsymbol{n}|_{\infty}>N, l \leq n_{j} \leq N\right\}, \quad \Lambda_{N}^{2, j}:=\left\{\boldsymbol{n} \in \mathbb{N}_{0}^{d}:|\boldsymbol{n}|_{\infty}>N, n_{j}>N\right\}$.

Now, we deal with the first summation. Clearly, for any $\boldsymbol{n} \in \Lambda_{N}^{1, j}$, there exists at least one index $k(k \neq j)$ such that $n_{k}>N$, so we obtain from (2.19) that

$$
\begin{aligned}
\sum_{\boldsymbol{n} \in \Lambda_{N}^{1, j}} \chi_{n_{j}, l}^{\alpha_{j}, \beta_{j}}\left|\hat{u}_{\boldsymbol{n}}^{\boldsymbol{\alpha}, \boldsymbol{\beta}}\right|^{2} & \leq \max _{\boldsymbol{n} \in \Lambda_{N}^{1, j}}\left\{\frac{\chi_{n_{j}, l}^{\alpha_{j}, \beta_{j}}}{\chi_{n_{k}, \beta_{k}}^{\alpha_{k}, \beta_{k}}}\right\} \sum_{\boldsymbol{n} \in \Lambda_{N}^{1, j}} \chi_{n_{k}, m}^{\alpha_{k}, \beta_{k}}\left|\hat{u}_{\boldsymbol{n}}^{\boldsymbol{\alpha}, \boldsymbol{\beta}}\right|^{2} \\
& \leq \max _{\boldsymbol{n} \in \Lambda_{N}^{1, j}}\left\{\frac{\chi_{n_{j}, l}^{\alpha_{j}, \beta_{j}}}{\chi_{n_{k}, \beta_{k}}^{\alpha_{k}, \beta_{k}}}\right\}\left\|\partial_{x_{k}}^{m} u\right\|_{\boldsymbol{\omega}^{\boldsymbol{\alpha}+m \boldsymbol{e}_{k}, \boldsymbol{\beta}+m \boldsymbol{e}_{k}, I^{d}}}^{2} \\
& \leq \frac{\chi_{N, l}^{\alpha_{j}, \beta_{j}}}{\chi_{N+1, m}^{\alpha_{k}, \beta_{k}}}\left\|\partial_{x_{k}}^{m} u\right\|_{\boldsymbol{\omega}^{\boldsymbol{\alpha}+m \boldsymbol{e}_{k}, \boldsymbol{\beta}+m \boldsymbol{e}_{k}, I^{d}} .}^{2} .
\end{aligned}
$$

Similarly, we treat the second summation in (2.27) as

$$
\begin{aligned}
& \sum_{\boldsymbol{n} \in \Lambda_{N}^{2, j}} \chi_{n_{j}, l}^{\alpha_{j}, \beta_{j}}\left|\hat{u}_{\boldsymbol{n}}^{\boldsymbol{\alpha}, \boldsymbol{\beta}}\right|^{2} \leq \max _{\boldsymbol{n} \in \Lambda_{N}^{2, j}}\left\{\frac{\chi_{n_{j}, l}^{\alpha_{j}, \beta_{j}}}{\chi_{n_{j}, m}^{\alpha_{j}, \beta_{j}}}\right\} \sum_{\boldsymbol{n} \in \Lambda_{N}^{2, j}} \chi_{n_{j}, m}^{\alpha_{j}, \beta_{j}}\left|\hat{u}_{\boldsymbol{n}}^{\boldsymbol{\alpha}, \boldsymbol{\beta}}\right|^{2} \\
& \leq \frac{\chi_{N+1, l}^{\alpha_{j}, \beta_{j}}}{\chi_{N+1, m}^{\alpha_{j}, \beta_{j}}}\left\|\partial_{x_{j}}^{m} u\right\|_{\boldsymbol{\omega}^{\alpha+m e_{j}, \boldsymbol{\beta}+m e_{j}, I^{d}}}^{2} .
\end{aligned}
$$

To obtain an explicit bound of the constants in terms of $N$, we recall Stirling's formula (cf. [9]):

$$
\Gamma(z)=\sqrt{2 \pi} z^{z-1 / 2} e^{-z}\left(1+O\left(z^{-1}\right)\right) \quad \forall z \gg 1,
$$

and we find from (2.10) that

$$
\chi_{n, k}^{a, b} \simeq n^{2 k} \quad \text { for } n \gg 1, k \geq 0, a, b>-1 .
$$

Copyright $@$ by SIAM. Unauthorized reproduction of this article is prohibited. 
Therefore, for any $1 \leq j \leq d$,

$$
\begin{aligned}
& \left\|\partial_{x_{j}}^{l}\left(\boldsymbol{\pi}_{N}^{\boldsymbol{\alpha}, \boldsymbol{\beta}} u-u\right)\right\|_{\boldsymbol{\omega}^{\alpha+l e_{j}, \boldsymbol{\beta}+l e_{j}, I^{d}}}^{2} \leq \hat{c} N^{2(l-m)}\left(\left\|\partial_{x_{j}}^{m} u\right\|_{\boldsymbol{\omega}^{\boldsymbol{\alpha}+m e_{j}, \boldsymbol{\beta}+m e_{j}, I^{d}}}^{2}\right. \\
& \left.+\left\|\partial_{x_{k}}^{m} u\right\|_{\boldsymbol{\omega}^{\boldsymbol{\alpha + m} \boldsymbol{e}_{k}, \boldsymbol{\beta}+m \boldsymbol{e}_{k}, I^{d}}}^{2}\right),
\end{aligned}
$$

where $\hat{c} \simeq 1$. By the definition (2.25), summing $1 \leq j \leq d$ leads to the desired result.

Remark 2.1. We note that error estimates for the full grid approximations have been derived previously by many authors (cf., for instance, $[6,8]$ ). However, the proof given above appears to be new. It is also much simpler and leads to more precise results in both norms on the left- and right-hand sides of (2.26).

We observe that the estimate on the full grid suffers from the curse of dimensionality, as the error decay rate with respect to $M=N^{d}$ deteriorates rapidly as $d$ increases. To circumvent such a curse, we study below the so-called hyperbolic cross approximations (cf. [21] and the references therein).

2.3.2. Hyperbolic cross Jacobi approximations. We now consider the finitedimensional space $X_{N}^{\boldsymbol{\alpha}, \boldsymbol{\beta}}$ corresponding to the hyperbolic cross index set:

$$
\Upsilon_{N}:=\Upsilon_{N}^{H}=\left\{\boldsymbol{n} \in \mathbb{N}_{0}^{d}: 1 \leq|\boldsymbol{n}|_{\text {mix }}:=\prod_{j=1}^{d} \max \left\{1, n_{j}\right\} \leq N\right\},
$$

namely,

$$
X_{N}^{\boldsymbol{\alpha}, \boldsymbol{\beta}}:=\operatorname{span}\left\{\boldsymbol{J}_{\boldsymbol{n}}^{\boldsymbol{\alpha}, \boldsymbol{\beta}}: \boldsymbol{n} \in \Upsilon_{N}\right\} .
$$

For convenience, we denote the $\boldsymbol{k}$-complement of $\Upsilon_{N}$ in (2.33) by

$$
\Upsilon_{N, \boldsymbol{k}}^{c}=\left\{\boldsymbol{n} \in \mathbb{N}_{0}^{d}:|\boldsymbol{n}|_{\text {mix }}>N \text { and } \boldsymbol{n} \geq \boldsymbol{k}\right\} \quad \forall \boldsymbol{k} \in \mathbb{N}_{0}^{d} .
$$

To illustrate the distribution and sparsity of the grids in $\Upsilon_{N}^{H}$, we plot in Figure 2.1 the hyperbolic cross $\Upsilon_{32}^{H}$ with $d=2$ (left) and $d=3$ (right).
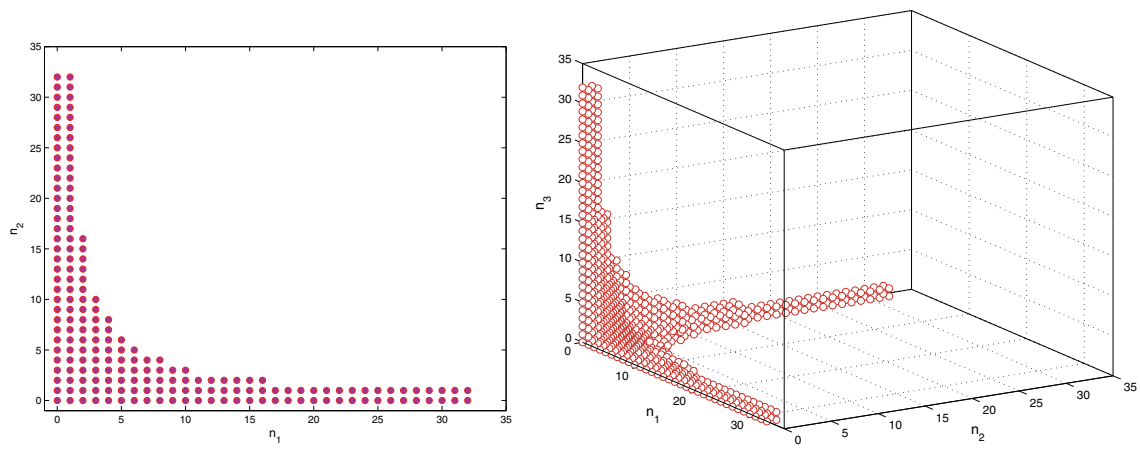

FIG. 2.1. The hyperbolic cross $\Upsilon_{32}^{H}$ with $d=2$ (left) and $d=3$ (right).

The following estimate on the cardinality of $\Upsilon_{N}^{H}$ can be found in, e.g., [11] and [15].

LEMMA 2.1.

$$
\operatorname{card}\left(\Upsilon_{N}^{H}\right)=C_{d} N(\ln N)^{d-1},
$$

where the constant $C_{d}$ depends on the dimension $d$. 
Remark 2.2. To demonstrate the dependence of $C_{d}$ on $d$, we plot in Figure 2.2 $C_{d}=\frac{\operatorname{card}\left(\Upsilon_{N}^{H}\right)}{N(\ln N)^{d-1}}$ for various $N \in[24,128]$ and $d \in[2,16]$, which indicates that $C_{d}$ is uniformly bounded and becomes smaller as $d$ increases. However, we do not know any rigorous proof for this fact.

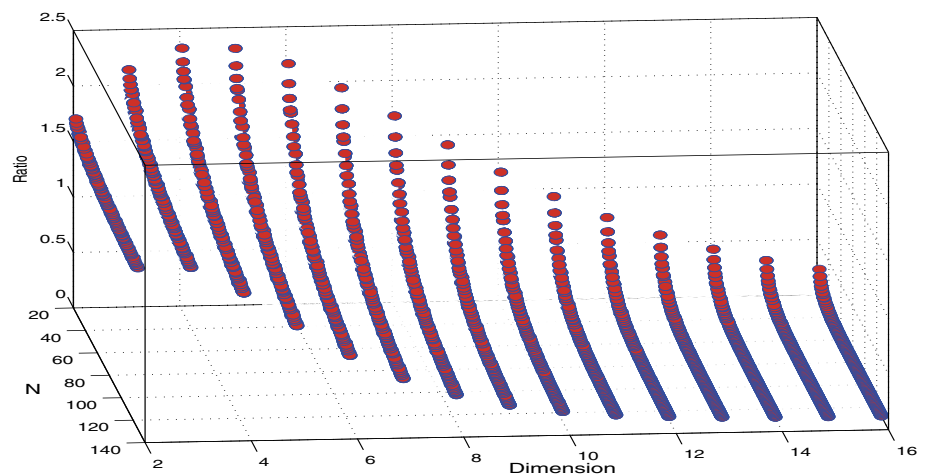

Fig. 2.2. The ratio $\operatorname{card}\left(\Upsilon_{N}^{H}\right) /\left(N(\ln N)^{d-1}\right)$ against various $N \in[24,128]$ and $d \in[2,16]$.

We now turn to the estimation of the truncation error $u-\pi_{N}^{\boldsymbol{\alpha}, \boldsymbol{\beta}} u$ of the hyperbolic cross approximation. In contrast with the Sobolev-type space (2.24) for the full grid, a suitable function space to characterize the hyperbolic cross approximation is the Jacobi-weighted Korobov-type space,

$$
\mathcal{K}_{\boldsymbol{\alpha}, \boldsymbol{\beta}}^{m}\left(I^{d}\right):=\left\{u: \boldsymbol{\partial}_{\boldsymbol{x}}^{\boldsymbol{k}} u \in L_{\boldsymbol{\omega}^{\alpha+\boldsymbol{k}, \boldsymbol{\beta}+\boldsymbol{k}}}^{2}\left(I^{d}\right), 0 \leq|\boldsymbol{k}|_{\infty} \leq m\right\} \quad \forall m \in \mathbb{N}_{0},
$$

with the norm and seminorm

$$
\begin{aligned}
& \|u\|_{\mathcal{K}_{\boldsymbol{\alpha}, \boldsymbol{\beta}}^{m}\left(I^{d}\right)}=\left(\sum_{0 \leq|\boldsymbol{k}|_{\infty} \leq m}\left\|\boldsymbol{\partial}_{\boldsymbol{x}}^{\boldsymbol{k}} u\right\|_{\boldsymbol{\omega}^{\alpha+\boldsymbol{k}, \boldsymbol{\beta}+\boldsymbol{k}, I^{d}}}^{2}\right)^{\frac{1}{2}}, \\
& |u|_{\mathcal{K}_{\boldsymbol{\alpha}, \boldsymbol{\beta}}^{m}\left(I^{d}\right)}=\left(\sum_{|\boldsymbol{k}|_{\infty}=m}\left\|\boldsymbol{\partial}_{\boldsymbol{x}}^{\boldsymbol{k}} u\right\|_{\boldsymbol{\omega}^{\alpha+\boldsymbol{k}, \boldsymbol{\beta}+\boldsymbol{k}, I^{d}}}^{2}\right)^{\frac{1}{2}} .
\end{aligned}
$$

Note the difference of the above definitions with those in (2.25). It is clear that $\mathcal{K}_{\boldsymbol{\alpha}, \boldsymbol{\beta}}^{0}\left(I^{d}\right)=L_{\boldsymbol{\omega}^{\boldsymbol{\alpha}, \boldsymbol{\beta}}}^{2}\left(I^{d}\right)$, and

$$
\mathcal{B}_{\boldsymbol{\alpha}, \boldsymbol{\beta}}^{d m}\left(I^{d}\right) \subset \mathcal{K}_{\boldsymbol{\alpha}, \boldsymbol{\beta}}^{m}\left(I^{d}\right) \subset \mathcal{B}_{\boldsymbol{\alpha}, \boldsymbol{\beta}}^{m}\left(I^{d}\right) .
$$

By virtue of (2.19), the norm and seminorm of $\mathcal{K}_{\boldsymbol{\alpha}, \boldsymbol{\beta}}^{m}\left(I^{d}\right)$ can be characterized in terms of the Jacobi expansion coefficients in (2.18):

$$
\begin{aligned}
& \|u\|_{\mathcal{K}_{\boldsymbol{\alpha}, \boldsymbol{\beta}}^{m}\left(I^{d}\right)}=\left\{\sum_{\boldsymbol{n} \in \mathbb{N}_{0}^{d}}\left(\sum_{0 \leq|\boldsymbol{k}|_{\infty} \leq m} \chi_{\boldsymbol{n}, \boldsymbol{k}}^{\boldsymbol{\alpha}, \boldsymbol{\beta}}\right)\left|\hat{u}_{\boldsymbol{n}}^{\boldsymbol{\alpha}, \boldsymbol{\beta}}\right|^{2}\right\}^{\frac{1}{2}}, \\
& |u|_{\mathcal{K}_{\boldsymbol{\alpha}, \boldsymbol{\beta}}^{m}\left(I^{d}\right)}=\left\{\sum_{\boldsymbol{n} \in \mathbb{N}_{0}^{d}}\left(\sum_{|\boldsymbol{k}|_{\infty}=m} \chi_{\boldsymbol{n}, \boldsymbol{k}}^{\boldsymbol{\alpha}, \boldsymbol{\beta}}\right)\left|\hat{u}_{\boldsymbol{n}}^{\boldsymbol{\alpha}, \boldsymbol{\beta}}\right|^{2}\right\}^{\frac{1}{2}} .
\end{aligned}
$$

Copyright $@$ by SIAM. Unauthorized reproduction of this article is prohibited. 
Thus, the space $\mathcal{K}_{\boldsymbol{\alpha}, \boldsymbol{\beta}}^{m}\left(I^{d}\right)$ can be viewed as an extension of the periodic Korobov space (1.6) for Fourier series to the Jacobi polynomial case.

The main result on the Jacobi hyperbolic cross approximation is stated below.

TheOrem 2.2. For any $u \in \mathcal{K}_{\boldsymbol{\alpha}, \boldsymbol{\beta}}^{m}\left(I^{d}\right)$,

$$
\left\|\boldsymbol{\partial}_{\boldsymbol{x}}^{\boldsymbol{l}}\left(\pi_{N}^{\boldsymbol{\alpha}, \boldsymbol{\beta}} u-u\right)\right\|_{\boldsymbol{\omega}^{l+\boldsymbol{\alpha}, \boldsymbol{l + \beta}, I^{d}}} \leq D_{1} N^{\left.|l|\right|_{\infty}-m}|u|_{\mathcal{K}_{\boldsymbol{\alpha}, \boldsymbol{\beta}}^{m}\left(I^{d}\right)}, \quad 0 \leq \boldsymbol{l} \leq m
$$

where $D_{1}=1$ for $m=0$, and for $m \geq 1$,

$$
D_{1}:=D_{1}(\boldsymbol{l}, m, d, \boldsymbol{\alpha}, \boldsymbol{\beta})=m^{(d-1)\left(m-|\boldsymbol{l}|_{\infty}\right)} \prod_{j=1}^{d}\left(\max \left\{1, \frac{m^{2}}{2 m+\alpha_{j}+\beta_{j}}\right\}\right)^{m-l_{j}} .
$$

Proof. Since the result is trivial for $m=0$, we assume $m \geq 1$.

By (2.17)-(2.19) and (2.22),

$$
\begin{aligned}
&\left\|\boldsymbol{\partial}_{\boldsymbol{x}}^{l}\left(\boldsymbol{\pi}_{N}^{\boldsymbol{\alpha}, \boldsymbol{\beta}} u-u\right)\right\|_{\boldsymbol{\omega}^{\alpha+l, \boldsymbol{\beta}+l, I^{d}}}^{2}=\sum_{\boldsymbol{n} \in \Upsilon_{N, l}^{c}} \chi_{\boldsymbol{n}, l}^{\boldsymbol{\alpha}, \boldsymbol{\beta}}\left|\hat{u}_{\boldsymbol{n}}^{\boldsymbol{\alpha}, \boldsymbol{\beta}}\right|^{2} \\
&=\sum_{\boldsymbol{n} \in \Upsilon_{N, m}^{c}} \chi_{\boldsymbol{n}, l}^{\boldsymbol{\alpha}, \boldsymbol{\beta}}\left|\hat{u}_{\boldsymbol{n}}^{\boldsymbol{\alpha}, \boldsymbol{\beta}}\right|^{2}+\sum_{\boldsymbol{n} \in \Upsilon_{N, l}^{c} \backslash \Upsilon_{N, m}^{c}} \chi_{\boldsymbol{n}, \boldsymbol{l}}^{\boldsymbol{\alpha}, \boldsymbol{\beta}}\left|\hat{u}_{\boldsymbol{n}}^{\boldsymbol{\alpha}, \boldsymbol{\beta}}\right|^{2} .
\end{aligned}
$$

Case (i) $\boldsymbol{n} \in \Upsilon_{N, m}^{c}$. In this case, $\boldsymbol{n} \geq m$, so we have

$$
\begin{aligned}
\sum_{\boldsymbol{n} \in \Upsilon_{N, m}^{c}} \chi_{\boldsymbol{n}, \boldsymbol{l}}^{\boldsymbol{\alpha}, \boldsymbol{\beta}}\left|\hat{u}_{\boldsymbol{n}}^{\boldsymbol{\alpha}, \boldsymbol{\beta}}\right|^{2} & \leq \max _{\boldsymbol{n} \in \Upsilon_{N, m}^{c}}\left\{\frac{\chi_{\boldsymbol{n}, \boldsymbol{l}}^{\boldsymbol{\alpha}, \boldsymbol{\beta}}}{\chi_{\boldsymbol{n}, m}^{\boldsymbol{\alpha}, \boldsymbol{\beta}}}\right\} \sum_{\boldsymbol{n} \in \Upsilon_{N, m}^{c}} \chi_{\boldsymbol{n}, m}^{\boldsymbol{\alpha}, \boldsymbol{\beta}}\left|\hat{u}_{\boldsymbol{n}}^{\boldsymbol{\alpha}, \boldsymbol{\beta}}\right|^{2} \\
& \stackrel{(2.19) \max _{\boldsymbol{n} \in \Upsilon_{N, m}^{c}}\left\{\frac{\boldsymbol{\chi}_{\boldsymbol{n}, \boldsymbol{l}}^{\boldsymbol{\alpha}, \boldsymbol{\beta}}}{\chi_{\boldsymbol{n}, m}^{\boldsymbol{\alpha}, \boldsymbol{\beta}}}\right\}\left\|\boldsymbol{\partial}_{\boldsymbol{x}}^{\boldsymbol{m}} u\right\|_{\boldsymbol{\omega}}^{\alpha+m, \boldsymbol{\beta}+m, I^{d}}}{2},
\end{aligned}
$$

where we have set $\boldsymbol{m}=(m, m, \ldots, m)$ and $\boldsymbol{\partial}_{\boldsymbol{x}}^{\boldsymbol{m}} u=\boldsymbol{\partial}_{\boldsymbol{x}}^{(m, m, \ldots, m)} u$. Thus, we only need to estimate the maximum value in (2.44).

A direct calculation by using (2.10) and (2.16) yields

$$
\begin{aligned}
\frac{\boldsymbol{\chi}_{\boldsymbol{n}, \boldsymbol{l}}^{\boldsymbol{\alpha}, \boldsymbol{\beta}}}{\boldsymbol{\chi}_{\boldsymbol{n}, \boldsymbol{\beta}}^{\boldsymbol{\beta}}} & =\prod_{j=1}^{d} \prod_{i=l_{j}}^{m-1} n_{j}^{-2}\left(1+\frac{\alpha_{j}+\beta_{j}+1}{n_{j}}-\frac{i\left(i+\alpha_{j}+\beta_{j}+1\right)}{n_{j}^{2}}\right)^{-1} \\
& =\left(\prod_{j=1}^{d} n_{j}^{2\left(l_{j}-m\right)}\right) \prod_{j=1}^{d} \prod_{i=l_{j}}^{m-1} \underbrace{\left(1+\frac{\alpha_{j}+\beta_{j}+1}{n_{j}}-\frac{i\left(i+\alpha_{j}+\beta_{j}+1\right)}{n_{j}^{2}}\right)^{-1}}_{:=g(i, j)} .
\end{aligned}
$$

Notice that for any $\boldsymbol{n} \in \Upsilon_{N, m}^{c}$ and $0 \leq|\boldsymbol{l}| \leq m$,

$$
\prod_{j=1}^{d} n_{j}^{2\left(l_{j}-m\right)} \leq \prod_{j=1}^{d} n_{j}^{2\left(|l|_{\infty}-m\right)} \leq N^{2\left(|l|_{\infty}-m\right)} .
$$

Copyright $@$ by SIAM. Unauthorized reproduction of this article is prohibited. 
Next, we estimate the upper bound of the second product in $(2.45)$. Note that $g(i, j)$ is decreasing with respect to $i$, i.e., $g(i, j) \leq g(m-1, j)$. Hence,

$$
\begin{aligned}
\prod_{j=1}^{d} \prod_{i=l_{j}}^{m-1} g(i, j) & \leq \prod_{j=1}^{d}[g(m-1, j)]^{m-l_{j}} \\
& =\prod_{j=1}^{d}\left(1+\frac{\alpha_{j}+\beta_{j}+1}{n_{j}}-\frac{(m-1)\left(m+\alpha_{j}+\beta_{j}+1\right)}{n_{j}^{2}}\right)^{l_{j}-m} .
\end{aligned}
$$

To obtain an upper bound independent of $N$, we define

$$
f_{j}(t):=-(m-1)\left(m+\alpha_{j}+\beta_{j}+1\right) t^{2}+\left(\alpha_{j}+\beta_{j}+1\right) t+1 \quad \text { with } \quad t=\frac{1}{n_{j}} .
$$

Assuming that $n_{1} n_{2} \cdots n_{d}=\tilde{N}>N \gg 1$, one verifies that

$$
m \leq n_{j} \leq \frac{\widetilde{N}}{m^{d-1}} \quad \Longrightarrow \quad \frac{m^{d-1}}{\widetilde{N}} \leq t \leq \frac{1}{m} .
$$

Obviously, for $m=1$, we have

$$
f_{j}(t)=1+\frac{\alpha_{j}+\beta_{j}+1}{n_{j}} \geq \begin{cases}1 & \text { if } \quad \alpha_{j}+\beta_{j}+1 \geq 0, \\ \alpha_{j}+\beta_{j}+2 & \text { if } \quad-2<\alpha_{j}+\beta_{j}+1<0 .\end{cases}
$$

For $m \geq 2$, using the properties of quadratic functions, we find that

$$
f_{j}(t) \geq \min \left\{f_{j}\left(\frac{1}{m}\right), f_{j}\left(\frac{m^{d-1}}{\widetilde{N}}\right)\right\} .
$$

A direct calculation leads to

$$
f_{j}\left(\frac{1}{m}\right)=\frac{2 m+\alpha_{j}+\beta_{j}}{m^{2}} ; \quad f_{j}\left(\frac{m^{d-1}}{\widetilde{N}}\right) \simeq 1 \quad \text { for } \tilde{N}>N \gg 1 .
$$

A combination of the above facts gives

$$
\prod_{j=1}^{d} \prod_{i=l_{j}}^{m-1} g(i, j) \leq \prod_{j=1}^{d}\left(\max \left\{1, \frac{m^{2}}{2 m+\alpha_{j}+\beta_{j}}\right\}\right)^{m-l_{j}}:=\tilde{c}^{2}
$$

which is valid for all $\boldsymbol{n} \geq m \geq 1$ and $N \gg 1$. Consequently, we derive from (2.45), (2.46), and (2.51) that

$$
\max _{\boldsymbol{n} \in \Upsilon_{N, m}^{\mathrm{c}}}\left\{\frac{\boldsymbol{\chi}_{\boldsymbol{n}, \boldsymbol{l}}^{\boldsymbol{\alpha}, \boldsymbol{\beta}}}{\chi_{\boldsymbol{n}, m}^{\boldsymbol{\alpha}, \boldsymbol{\beta}}}\right\} \leq \tilde{c}^{2} N^{2\left(|\boldsymbol{l}|_{\infty}-m\right)}
$$

Now, we deal with the second summation in (2.43). 
Case (ii) $\boldsymbol{n} \in \Upsilon_{N, \boldsymbol{l}}^{c} \backslash \Upsilon_{N, m}^{c}$. In this case, a little care has to be taken for the modes with $n_{j}<m$. Notice that

$$
\Upsilon_{N, \boldsymbol{l}}^{c} \backslash \Upsilon_{N, m}^{c}=\left\{\boldsymbol{n} \in \mathbb{N}_{0}^{d}:|\boldsymbol{n}|_{\text {mix }}>N, \boldsymbol{n} \geq \boldsymbol{l}, \exists j, \text { s.t. } l_{j} \leq n_{j}<m\right\} .
$$

For clarity, we split the index set $\{1 \leq j \leq d\}=\aleph \cup \aleph^{c}$ with

$$
\aleph=\left\{j: l_{j} \leq n_{j}<m, 1 \leq j \leq d\right\}, \quad \aleph^{c}=\left\{j: n_{j} \geq m, 1 \leq j \leq d\right\} .
$$

Clearly, $\aleph \cap \aleph^{c}=\varnothing$, and neither of these two index sets is empty. Define

$$
\tilde{\chi}_{n_{j}, l_{j}, m}^{\alpha_{j}, \beta_{j}}:=\max \left\{\chi_{n_{j}, l_{j}}^{\alpha_{j}, \beta_{j}}, \chi_{n_{j}, m}^{\alpha_{j}, \beta_{j}}\right\}= \begin{cases}0 & \text { if } n_{j}<l_{j}, \\ \chi_{n_{j}, l_{j}}^{\alpha_{j}, \beta_{j}} & \text { if } l_{j} \leq n_{j}<m, \\ \chi_{n_{j}, m_{j}}^{\alpha_{j}, \beta_{j}} & \text { if } m \leq n_{j} .\end{cases}
$$

Hence, for any $j \in \aleph, \tilde{\chi}_{n_{j}, l_{j}, m}^{\alpha_{j}, \beta_{j}}=\chi_{n_{j}, l_{j}}^{\alpha_{j}, \beta_{j}}$, while for any $j \in \aleph^{c}, \tilde{\chi}_{n_{j}, l_{j}, m}^{\alpha_{j}, \beta_{j}}=\chi_{n_{j}, m}^{\alpha_{j}, \beta_{j}}$. Moreover,

$$
\widetilde{\boldsymbol{\chi}}_{\boldsymbol{n}, \boldsymbol{l}, m}^{\boldsymbol{\alpha}, \boldsymbol{\beta}}=\left(\prod_{j \in \aleph} \chi_{n_{j}, l_{j}}^{\alpha_{j}, \beta_{j}}\right)\left(\prod_{k \in \aleph^{c}} \chi_{n_{k}, \beta_{k}}^{\alpha_{k}, \beta_{k}}\right)=\chi_{\boldsymbol{n}, \boldsymbol{k}}^{\boldsymbol{\alpha}, \boldsymbol{\beta}},
$$

where $\boldsymbol{k}$ is a $d$-dimensional index consisting of $l_{j}$ for $j \in \aleph$ and $m$ for $j \in \aleph^{c}$. Since $|\boldsymbol{k}|_{\infty}=m$, we find from (2.19) and (2.40) that

$$
\sum_{\boldsymbol{n} \in \Upsilon_{N, l}^{c} \backslash \Upsilon_{N, m}^{c}} \widetilde{\chi}_{\boldsymbol{n}, \boldsymbol{l}, m}^{\boldsymbol{\alpha}, \boldsymbol{\beta}}\left|\hat{u}_{\boldsymbol{n}}^{\boldsymbol{\alpha}, \boldsymbol{\beta}}\right|^{2}=\sum_{\boldsymbol{n} \in \Upsilon_{N, l}^{c} \backslash \Upsilon_{N, m}^{c}} \chi_{\boldsymbol{n}, \boldsymbol{k}}^{\boldsymbol{\alpha}, \boldsymbol{\beta}}\left|\hat{u}_{\boldsymbol{n}}^{\boldsymbol{\alpha}, \boldsymbol{\beta}}\right|^{2} \leq|u|_{\mathcal{K}_{\boldsymbol{\alpha}, \boldsymbol{\beta}}^{m}\left(I^{d}\right)}^{2} .
$$

We treat the second summation in (2.43) as

$$
\begin{aligned}
& \sum_{\boldsymbol{n} \in \Upsilon_{N, l}^{c} \backslash \Upsilon_{N, m}^{c}} \chi_{\boldsymbol{n}, l}^{\boldsymbol{\alpha}, \boldsymbol{\beta}}\left|\hat{u}_{\boldsymbol{n}}^{\boldsymbol{\alpha}, \boldsymbol{\beta}}\right|^{2}=\max _{\boldsymbol{n} \in \Upsilon_{N, l}^{c} \backslash \Upsilon_{N, m}^{c}}\left\{\frac{\boldsymbol{\chi}_{\boldsymbol{n}, \boldsymbol{l}}^{\boldsymbol{\alpha}, \boldsymbol{\beta}}}{\tilde{\chi}_{\boldsymbol{n}, \boldsymbol{l}, m}^{\boldsymbol{\alpha}, \boldsymbol{\beta}}}\right\} \sum_{\boldsymbol{n} \in \Upsilon_{N, l}^{c} \backslash \Upsilon_{N, m}^{c}} \widetilde{\chi}_{\boldsymbol{n}, \boldsymbol{l}, m}^{\boldsymbol{\alpha}, \boldsymbol{\beta}}\left|\hat{u}_{\boldsymbol{n}}^{\boldsymbol{\alpha}, \boldsymbol{\beta}}\right|^{2} \\
& \stackrel{(2.56)}{\leq} \max _{\boldsymbol{n} \in \Upsilon_{N, l}^{c} \backslash \Upsilon_{N, m}^{c}}\left\{\frac{\boldsymbol{\chi}_{\boldsymbol{n}, \boldsymbol{l}}^{\boldsymbol{\alpha}, \boldsymbol{\beta}}}{\widetilde{\boldsymbol{\chi}} \boldsymbol{n}, \boldsymbol{l}, m_{\boldsymbol{\alpha}, \boldsymbol{\beta}}}\right\}|u|_{\mathcal{K}_{\boldsymbol{\alpha}, \boldsymbol{\beta}}^{m}\left(I^{d}\right)}^{2} .
\end{aligned}
$$

Thus, it remains to estimate the maximum. By a direct calculation,

$$
\begin{aligned}
\frac{\boldsymbol{\chi}_{\boldsymbol{n}, \boldsymbol{l}}^{\boldsymbol{\alpha}, \boldsymbol{\beta}}}{\widetilde{\boldsymbol{\chi}}_{\boldsymbol{n}, \boldsymbol{l}, m}^{\boldsymbol{\alpha}, \boldsymbol{\beta}}} & =\prod_{j \in \aleph^{c}} \frac{\chi_{n_{j}, l_{j}}^{\alpha_{j}, \beta_{j}}}{\chi_{n_{j}, \beta_{j}}^{\alpha_{j},,_{j}}} \leq\left(\prod_{j \in \aleph^{c}} n_{j}^{2\left(l_{j}-m\right)}\right) \prod_{j \in \aleph^{c}} \prod_{i=l_{j}}^{m-1} g(i, j) \\
& \leq\left(\prod_{j \in \aleph^{c}} n_{j}^{2\left(l_{j}-m\right)}\right) \prod_{j \in \aleph^{c}}\left(\max \left\{1, \frac{m^{2}}{2 m+\alpha_{j}+\beta_{j}}\right\}\right)^{m-l_{j}} \\
& \stackrel{(2.51)}{\leq} \tilde{c}^{2}\left(\prod_{j \in \aleph^{c}} n_{j}^{2\left(l_{j}-m\right)}\right) .
\end{aligned}
$$

In view of $m \geq 1$ and $|\boldsymbol{n}|_{\text {mix }}=\bar{n}_{1} \cdots \bar{n}_{d}>N$, we deduce that

$$
\prod_{j \in \aleph^{c}} \bar{n}_{j}>\frac{N}{\prod_{j \in \aleph} \bar{n}_{j}}>\frac{N}{\prod_{j \in \aleph} m} .
$$

Copyright $@$ by SIAM. Unauthorized reproduction of this article is prohibited. 
A combination of the above estimates leads to

$$
\begin{aligned}
\frac{\boldsymbol{\chi}_{\boldsymbol{n}, \boldsymbol{l}}^{\boldsymbol{\alpha}, \boldsymbol{\beta}}}{\widetilde{\boldsymbol{\chi}}_{\boldsymbol{n}, \boldsymbol{l}, \boldsymbol{\beta}}^{\boldsymbol{\alpha}, m}} & \leq \tilde{c}^{2} \prod_{j \in \aleph^{c}} n_{j}^{2\left(l_{j}-m\right)} \leq \tilde{c}^{2} \prod_{j \in \aleph^{c}} n_{j}^{2\left(|\boldsymbol{l}|_{\infty}-m\right)} \leq \tilde{c}^{2}\left(\frac{N}{\prod_{j \in \aleph} m}\right)^{2\left(|\boldsymbol{l}|_{\infty}-m\right)} \\
& \leq \tilde{c}^{2} m^{2(d-1)\left(m-|\boldsymbol{l}|_{\infty}\right)} N^{2\left(|\boldsymbol{l}|_{\infty}-m\right)} .
\end{aligned}
$$

Finally, the estimate (2.41)-(2.42) follows from (2.43), (2.52), (2.57), and (2.60).

By the definition of $\mathcal{K}_{\boldsymbol{\alpha}, \boldsymbol{\beta}}^{l}\left(I^{d}\right)$ in $(2.37)-(2.38)$ and Theorem 2.2, we immediately obtain the following result.

COROLlary 2.1.

$$
\left\|\boldsymbol{\pi}_{N}^{\boldsymbol{\alpha}, \boldsymbol{\beta}} u-u\right\|_{\mathcal{K}_{\boldsymbol{\alpha}, \boldsymbol{\beta}}^{l}\left(I^{d}\right)} \leq D_{2} N^{l-m}|u|_{\mathcal{K}_{\boldsymbol{\alpha}, \boldsymbol{\beta}}^{m}\left(I^{d}\right)}, \quad 0 \leq l \leq m,
$$

where the constant $D_{2}$ is given by

$$
D_{2}:=D_{2}(l, m, d, \boldsymbol{\alpha}, \boldsymbol{\beta})=\left(\sum_{0 \leq|\boldsymbol{l}|_{\infty} \leq l} D_{1}^{2} N^{2\left(|\boldsymbol{l}|_{\infty}-l\right)}\right)^{\frac{1}{2}}
$$

with $D_{1}$ being the same as in Theorem 2.2 .

The above result clearly indicates that the Jacobi-weighted Korobov-type spaces $\mathcal{K}_{\boldsymbol{\alpha}, \boldsymbol{\beta}}^{l}\left(I^{d}\right)$ are the natural functional spaces for hyperbolic cross approximations. We note that the above result is in the same form as the result in Theorem 2.1, except that Jacobi-weighted Korobov norms are used here instead of the Jacobi-weighted Sobolev norms used in Theorem 2.1.

To characterize the error in terms of the dimensionality of the approximation space $X_{N}^{\boldsymbol{\alpha}, \boldsymbol{\beta}}$, we find from Lemma 2.1 that for any $\varepsilon>0$, and for $N \gg 1$,

$$
M \leq C_{d} N^{1+\varepsilon(d-1)} \quad \Rightarrow \quad N^{-1} \leq C_{d}^{1 /(1+\varepsilon(d-1))} M^{-(1 /(1+\varepsilon(d-1)))} .
$$

Therefore, as a direct consequence of Corollary 2.1, we have the following estimate.

Corollary 2.2. For any $\varepsilon>0$ and $0 \leq l \leq m$,

$$
\left\|\boldsymbol{\pi}_{N}^{\boldsymbol{\alpha}, \boldsymbol{\beta}} u-u\right\|_{\mathcal{K}_{\boldsymbol{\alpha}, \boldsymbol{\beta}}^{l}\left(I^{d}\right)} \leq D_{2} C_{d}^{1 /(1+\varepsilon(d-1))} M^{\frac{\left.|l|\right|_{\infty}-m}{1+\varepsilon(d-1)}}|u|_{\mathcal{K}_{\boldsymbol{\alpha}, \boldsymbol{\beta}}^{m}\left(I^{d}\right)} .
$$

2.3.3. Optimized hyperbolic cross Jacobi approximations. While the use of the regular hyperbolic cross (2.34) significantly improved the convergence rate with respect to the number of unknowns, the curse of dimensionality is not completely broken as the convergence rate still deteriorates, albeit very slowly, as $d$ increases (cf. (2.63)). In order to completely break the curse of dimensionality, we consider the following family of spaces (cf. $[7,15])$ :

$$
V_{N, \gamma}^{\boldsymbol{\alpha}, \boldsymbol{\beta}}:=\operatorname{span}\left\{\boldsymbol{J}_{\boldsymbol{n}, \boldsymbol{\beta}}^{\boldsymbol{\alpha}}:|\boldsymbol{n}|_{\text {mix }}|\boldsymbol{n}|_{\infty}^{-\gamma} \leq N^{1-\gamma}\right\}, \quad-\infty \leq \gamma<1 .
$$

In particular, we have $V_{N, 0}^{\boldsymbol{\alpha}, \boldsymbol{\beta}}=X_{N}^{\boldsymbol{\alpha}, \boldsymbol{\beta}}$ in $(2.34)$ and $V_{N,-\infty}^{\boldsymbol{\alpha}, \boldsymbol{\beta}}:=\operatorname{span}\left\{\boldsymbol{J}_{\boldsymbol{n}}^{\boldsymbol{\alpha}, \boldsymbol{\beta}}:|\boldsymbol{n}|_{\infty} \leq N\right\}$ (i.e., the full grid in (2.23)). But for $0<\gamma<1$, the trade-off between $N^{\gamma}$ and $|\boldsymbol{n}|_{\infty}^{\gamma}$ leads to the following reduction of cardinality (see Lemma 3 in [15]):

$$
\operatorname{card}\left(V_{N, \gamma}^{\boldsymbol{\alpha}, \boldsymbol{\beta}}\right)=C(\gamma, d) N, \quad 0<\gamma<1 .
$$

Copyright $@$ by SIAM. Unauthorized reproduction of this article is prohibited. 


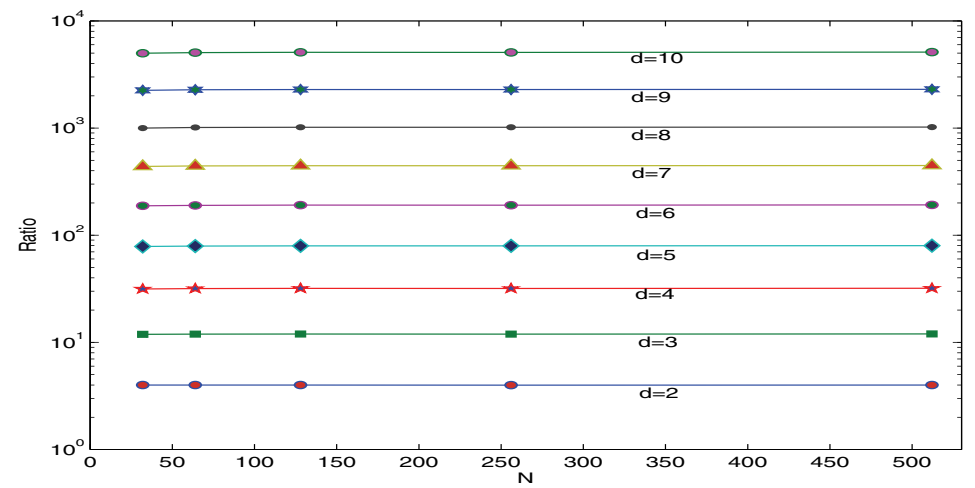

FIG. 2.3. The ratio $\operatorname{dim}\left(V_{N, \gamma}^{\boldsymbol{\alpha}, \boldsymbol{\beta}}\right) / N$ against various $N$ with $\gamma=0.9$ and $d=2,3, \ldots, 10$.

Thus, the space $V_{N, \gamma}^{\boldsymbol{\alpha}, \boldsymbol{\beta}}$ with $0<\gamma<1$ is referred as the optimized hyperbolic cross space.

We plot in Figure 2.3 the ratio $\operatorname{dim}\left(V_{N, \gamma}^{\boldsymbol{\alpha}, \boldsymbol{\beta}}\right) / N$ for various $N$ with $\gamma=0.9$ and $d=2, \ldots, 10$, which indicates that the constant $C(\gamma, d)$ is independent of $N$, but grows as $d$ increases.

In this case, the complement index set in (2.35) takes the form

$$
\Upsilon_{N, \boldsymbol{k}}^{c}=\left\{\boldsymbol{n} \in \mathbb{N}_{0}^{d}:|\boldsymbol{n}|_{\text {mix }}|\boldsymbol{n}|_{\infty}^{-\gamma}>N^{1-\gamma} \text { and } \boldsymbol{n} \geq \boldsymbol{k}\right\} \quad \forall \boldsymbol{k} \in \mathbb{N}_{0}^{d}
$$

The main approximation result based on the optimized hyperbolic cross is stated as follows.

TheOREM 2.3. For any $u \in \mathcal{K}_{\boldsymbol{\alpha}, \boldsymbol{\beta}}^{m}\left(I^{d}\right)$ and $0 \leq|\boldsymbol{l}|_{1} \leq m$,

$$
\begin{aligned}
\left\|\boldsymbol{\partial}_{\boldsymbol{x}}^{l}\left(\boldsymbol{\pi}_{N, \gamma}^{\boldsymbol{\alpha}, \boldsymbol{\beta}} u-u\right)\right\|_{\boldsymbol{\omega}^{\alpha+l, \boldsymbol{\beta}+l, I^{d}}} \leq D_{3}|u|_{\mathcal{K}_{\boldsymbol{\alpha}, \boldsymbol{\beta}}^{m}\left(I^{d}\right)} \\
\times \begin{cases}N^{|l|_{1}-m} & \text { if } 0<\gamma \leq \frac{|\boldsymbol{l}|_{1}}{m}, \\
N^{|l|_{1}-m+\left(\gamma m-\left.|l|\right|_{1}\right)\left(1-\frac{1}{d}\right)} & \text { if } \quad \frac{|\boldsymbol{l}|_{1}}{m} \leq \gamma<1,\end{cases}
\end{aligned}
$$

where

$$
\begin{aligned}
D_{3}:= & {\left[\prod_{j=1}^{d}\left(\max \left\{1, \frac{m^{2}}{2 m+\alpha_{j}+\beta_{j}}\right\}\right)^{m-l_{j}}\right] } \\
& \times m^{(d-1) m} \times \begin{cases}m^{-\frac{(d-1)\left(|l|_{1}-\gamma m\right)}{1-\gamma}} & \text { if } 0<\gamma \leq \frac{|l|_{1}}{m}, \\
1 & \text { if } \frac{|l|_{1}}{m} \leq \gamma<1 .\end{cases}
\end{aligned}
$$

Proof. The estimate is trivial for $m=0$, so we assume $m \geq 1$. Let $\widetilde{\boldsymbol{\chi}}_{\boldsymbol{n}, \boldsymbol{l}, \boldsymbol{\boldsymbol { \beta }}}^{\boldsymbol{\alpha}, \boldsymbol{\beta}}$ be the same as defined in (2.55). Following the proof of Theorem 2.2, we begin by estimating (2.43) with $\Upsilon_{N, l}^{c}$ defined in (2.66), and separated into two subsets: (i) $\Upsilon_{N, m}^{c}$ and (ii) $\Upsilon_{N, l}^{c} \backslash \Upsilon_{N, m}^{c}$ as before, namely,

$$
\begin{aligned}
& \left\|\boldsymbol{\partial}_{\boldsymbol{x}}^{l}\left(\boldsymbol{\pi}_{N}^{\boldsymbol{\alpha}, \boldsymbol{\beta}} u-u\right)\right\|_{\boldsymbol{\omega}^{\alpha+l, \boldsymbol{\beta}+l, I^{d}}}^{2} \leq \max _{\boldsymbol{n} \in \Upsilon_{N, m}^{c}}\left\{\frac{\boldsymbol{\chi}_{\boldsymbol{n}, l}^{\boldsymbol{\alpha}, \boldsymbol{\beta}}}{\widetilde{\chi}_{\boldsymbol{n}, \boldsymbol{l}, m}^{\boldsymbol{\alpha}, \boldsymbol{\beta}}}\right\} \sum_{\boldsymbol{n} \in \Upsilon_{N, m}^{c}} \widetilde{\chi}_{\boldsymbol{n}, \boldsymbol{l}, m}^{\boldsymbol{\alpha}, \boldsymbol{\beta}}\left|\hat{u}_{\boldsymbol{n}}^{\boldsymbol{\alpha}, \boldsymbol{\beta}}\right|^{2} \\
& +\max _{\boldsymbol{n} \in \Upsilon_{N, l}^{c} \backslash \Upsilon_{N, m}^{c}}\left\{\frac{\boldsymbol{\chi}_{\boldsymbol{n}, l}^{\boldsymbol{\alpha}, \boldsymbol{\beta}}}{\widetilde{\chi}_{\boldsymbol{n}, \boldsymbol{l}, m}^{\boldsymbol{\alpha}, \boldsymbol{\beta}}}\right\} \sum_{\boldsymbol{n} \in \Upsilon_{N, l}^{c} \backslash \Upsilon_{N, m}^{c}} \widetilde{\chi}_{\boldsymbol{n}, \boldsymbol{l}, m}^{\boldsymbol{\alpha}, \boldsymbol{\beta}}\left|\hat{u}_{\boldsymbol{n}}^{\boldsymbol{\alpha}, \boldsymbol{\beta}}\right|^{2} .
\end{aligned}
$$

Copyright $\odot$ by SIAM. Unauthorized reproduction of this article is prohibited. 
We now estimate the first term and consider $\boldsymbol{n} \in \Upsilon_{N, m}^{c}$. Like (2.45), we have

$$
\frac{\boldsymbol{\chi}_{\boldsymbol{n}, \boldsymbol{l}}^{\boldsymbol{\alpha}, \boldsymbol{\beta}}}{\boldsymbol{\chi}_{\boldsymbol{n}, \boldsymbol{\beta}}^{\boldsymbol{\alpha}, \boldsymbol{\beta}}}=\left(\prod_{j=1}^{d} n_{j}^{2\left(l_{j}-m\right)}\right)\left\{\prod_{j=1}^{d} \prod_{i=l_{j}}^{m-1} g(i, j)\right\} .
$$

We first deal with the product in parentheses. Notice that for any $\boldsymbol{n} \in \Upsilon_{N, m}^{c}$,

$$
|\boldsymbol{n}|_{\text {mix }}|\boldsymbol{n}|_{\infty}^{-\gamma}>N^{1-\gamma} \Longrightarrow\left(\frac{|\boldsymbol{n}|_{\infty}^{\gamma}}{|\boldsymbol{n}|_{\text {mix }}}\right)^{\frac{1}{1-\gamma}}<\frac{1}{N} .
$$

Therefore,

$$
\begin{gathered}
\prod_{j=1}^{d} n_{j}^{2\left(l_{j}-m\right)}=\left(\prod_{j=1}^{d} n_{j}^{2 l_{j}}\right)\left(\prod_{j=1}^{d} n_{j}\right)^{-2 m} \leq\left(\prod_{j=1}^{d}|\boldsymbol{n}|_{\infty}^{2 l_{j}}\right)|\boldsymbol{n}|_{\text {mix }}^{-2 m}=|\boldsymbol{n}|_{\infty}^{2|l|_{1}}|\boldsymbol{n}|_{\text {mix }}^{-2 m} \\
\quad=\left(\frac{|\boldsymbol{n}|_{\infty}^{\gamma}}{|\boldsymbol{n}|_{\text {mix }}}\right)^{\frac{2\left(m-\left.|l|\right|_{1}\right)}{1-\gamma}}\left(\frac{|\boldsymbol{n}|_{\infty}}{|\boldsymbol{n}|_{\text {mix }}}\right)^{\frac{2\left(\mid l l_{1}-\gamma m\right)}{1-\gamma}} \stackrel{(2.70)}{\leq} N^{2\left(|l|_{1}-m\right)}\left(\frac{|\boldsymbol{n}|_{\infty}}{|\boldsymbol{n}|_{\text {mix }}}\right)^{\frac{2\left(|l|_{1}-\gamma m\right)}{1-\gamma}}
\end{gathered}
$$

One verifies readily that

$$
\frac{|\boldsymbol{n}|_{\infty}}{|\boldsymbol{n}|_{\text {mix }}} \leq \frac{1}{m^{d-1}} \quad \forall \boldsymbol{n} \in \Upsilon_{N, m}^{c}
$$

Hence, if $0<\gamma \leq \frac{|l|_{1}}{m}$,

$$
\prod_{j=1}^{d} n_{j}^{2\left(l_{j}-m\right)} \leq N^{2\left(|\boldsymbol{l}|_{1}-m\right)} \max _{\boldsymbol{n} \in \Upsilon_{N, m}^{\mathrm{c}}}\left(\frac{|\boldsymbol{n}|_{\infty}}{|\boldsymbol{n}|_{\text {mix }}}\right)^{\frac{2\left(|l| l_{1}-\gamma m\right)}{1-\gamma}} \leq m^{-\frac{2(d-1)\left(|l|_{1}-\gamma m\right)}{1-\gamma}} N^{2\left(|\boldsymbol{l}|_{1}-m\right)} .
$$

Next, for any $\boldsymbol{n} \in \Upsilon_{N, m}^{c}$, we have $|\boldsymbol{n}|_{\infty}>N^{\frac{1}{d}}$ and

$$
|\boldsymbol{n}|_{\text {mix }}|\boldsymbol{n}|_{\infty}^{-\gamma}>N^{1-\gamma} \Rightarrow \frac{|\boldsymbol{n}|_{\text {mix }}}{|\boldsymbol{n}|_{\infty}}<N^{1-\gamma}|\boldsymbol{n}|_{\infty}^{\gamma-1}<N^{(1-\gamma)\left(1-\frac{1}{d}\right)},
$$

which, together with (2.71), implies that if $\frac{|l|_{1}}{m} \leq \gamma<1$,

$$
\prod_{j=1}^{d} n_{j}^{2\left(l_{j}-m\right)} \leq N^{2\left(\left.|l|\right|_{1}-m\right)} \max _{n \in \Upsilon_{N, m}^{c}}\left(\frac{|\boldsymbol{n}|_{\infty}}{|\boldsymbol{n}|_{\text {mix }}}\right)^{\frac{2\left(|l|_{1}-\gamma m\right)}{1-\gamma}} \leq N^{2\left(|l|_{1}-m\right)+2\left(\gamma m-|l|_{1}\right)\left(1-\frac{1}{d}\right)} .
$$

It remains to estimate the term in braces in (2.69). Observe that for any $\boldsymbol{n} \in \Upsilon_{N, m}^{c}$, we have

$$
|\boldsymbol{n}|_{\infty} \geq|\boldsymbol{n}|_{\text {mix }}^{\frac{1}{d}} \Rightarrow|\boldsymbol{n}|_{\text {mix }}>N^{(1-\gamma) /\left(1-\frac{\gamma}{d}\right)} \gg 1 .
$$

Hence, the product in braces with maximum taken over $\Upsilon_{N, m}^{c}$ (cf. (2.66)) has the same upper bound $\tilde{c}^{2}$ as in (2.51).

Copyright (c) by SIAM. Unauthorized reproduction of this article is prohibited. 
Next we consider the second summation in (2.68). Defining $\aleph^{c}$ and $\aleph^{c}$ associated with $\Upsilon_{N, \boldsymbol{l}}^{c} \backslash \Upsilon_{N, m}^{c}$ as in (2.53), and following the derivation of the estimate (2.60), we have

$$
\begin{aligned}
\frac{\boldsymbol{\chi}_{\boldsymbol{n}, \boldsymbol{l}}^{\boldsymbol{\alpha}, \boldsymbol{\beta}}}{\widetilde{\boldsymbol{\chi}}_{\boldsymbol{n}, \boldsymbol{l}, \boldsymbol{\beta}}^{\boldsymbol{\alpha}, \boldsymbol{\beta}}} & =\left(\prod_{j \in \aleph^{\prime}} \frac{\chi_{n_{j}, l_{j}}^{\alpha_{j}, \beta_{j}}}{\tilde{\chi}_{n_{j}, l_{j}, m}^{\alpha_{j}, \beta_{j}}}\right)\left(\prod_{k \in \aleph^{c}} \frac{\chi_{n_{k}, l_{k}}^{\alpha_{k}, \beta_{k}}}{\tilde{\chi}_{n_{k}, l_{k}, m}^{\alpha_{k}, \beta_{k}}}\right) \stackrel{(2.51)}{\leq} \tilde{c}^{2} \prod_{k \in \aleph^{c}} n_{k}^{2\left(l_{k}-m\right)} \\
& \leq \tilde{c}^{2}\left(\prod_{k \in \aleph^{c}}|\boldsymbol{n}|_{\infty}^{2 l_{k}}\right)\left(\frac{\prod_{j \in \aleph^{\prime}} \bar{n}_{j}}{|\boldsymbol{n}|_{\operatorname{mix}}}\right)^{2 m} \leq \tilde{c}^{2} m^{2(d-1) m}|\boldsymbol{n}|_{\infty}^{2|l| l_{1}}|\boldsymbol{n}|_{\text {mix }}^{-2 m} \\
& \stackrel{(2.71)}{\leq} \tilde{c}^{2} m^{2(d-1) m} N^{2\left(|l|_{1}-m\right)}\left(\frac{|\boldsymbol{n}|_{\infty}}{|\boldsymbol{n}|_{\text {mix }}}\right)^{\frac{2\left(\mid l l_{1}-\gamma m\right)}{1-\gamma}} .
\end{aligned}
$$

Since the estimate (2.72) is also valid for all $\boldsymbol{n} \in \Upsilon_{N, \boldsymbol{l}}^{c} \backslash \Upsilon_{N, m}^{c}$, we can follow the derivations of (2.73)-(2.75) to obtain

$$
\frac{\boldsymbol{\chi}_{\boldsymbol{n}, \boldsymbol{l}}^{\boldsymbol{\alpha}, \boldsymbol{\beta}}}{\widetilde{\boldsymbol{\chi}}_{\boldsymbol{n}, \boldsymbol{l}, m}^{\boldsymbol{\alpha}, \boldsymbol{\beta}}} \leq \tilde{c}^{2} m^{2(d-1) m} \times \begin{cases}m^{-\frac{2(d-1)\left(|\boldsymbol{l}|_{1}-\gamma m\right)}{1-\gamma}} N^{2\left(|\boldsymbol{l}|_{1}-m\right)} & \text { if } 0<\gamma \leq \frac{|\boldsymbol{l}|_{1}}{m} \\ N^{2\left(|\boldsymbol{l}|_{1}-m\right)+2\left(\gamma m-|\boldsymbol{l}|_{1}\right)\left(1-\frac{1}{d}\right)} & \text { if } \frac{|\boldsymbol{l}|_{1}}{m} \leq \gamma<1\end{cases}
$$

Furthermore, (2.56) holds for the optimized hyperbolic cross.

Finally, a combination of the above estimates leads to the desired result.

Unlike the results for the regular hyperbolic approximation in Theorem 2.2, we cannot replace the norm on the left-hand side of (2.67) by the norm in $\mathcal{K}_{\boldsymbol{\alpha}, \boldsymbol{\beta}}^{l}\left(I^{d}\right)$ as in Corollary 2.1, due to the term " $|\boldsymbol{l}|_{1}$ " in the power of $N$. Instead, we can derive immediately the following estimate in the weighted Sobolev space $\mathcal{B}_{\boldsymbol{\alpha}, \boldsymbol{\beta}}^{l}\left(I^{d}\right)$.

Corollary 2.3. For any $u \in \mathcal{K}_{\boldsymbol{\alpha}, \boldsymbol{\beta}}^{m}\left(I^{d}\right), 0 \leq l \leq m$, and $0<\gamma<1$,

$$
\left\|\boldsymbol{\pi}_{N, \gamma}^{\boldsymbol{\alpha}, \boldsymbol{\beta}} u-u\right\|_{\mathcal{B}_{\boldsymbol{\alpha}, \boldsymbol{\beta}}^{l}\left(I^{d}\right)} \leq D_{4} N^{l-m}|u|_{\mathcal{K}_{\boldsymbol{\alpha}, \boldsymbol{\beta}}^{m}\left(I^{d}\right)}, \quad 0<\gamma \leq \frac{l}{m},
$$

where

$$
D_{4}=\left(\sum_{0 \leq|l|_{1} \leq l} D_{3}^{2} N^{2\left(|l|_{1}-l\right)}\right)^{\frac{1}{2}}
$$

and $D_{3}$ is the same as in Theorem 2.3 .

The above result provides a convergence rate which is independent of dimension $d$ for the approximation space $V_{N, \gamma}^{\boldsymbol{\alpha}, \boldsymbol{\beta}}$.

2.4. Hyperbolic cross approximations by generalized Jacobi polynomials. As illustrated in [16, 17], the use of generalized Jacobi polynomials (GJPs) greatly simplifies the analysis and implementation of spectral methods. We now show that the results established in the previous subsection can be extended to the case of GJPs with both indexes being integers.

We first recall the definition of GJPs in [16]. Let $k, l \in \mathbb{Z}$ (the set of all integers) and define

$$
J_{n}^{k, l}(x)=\left\{\begin{array}{lll}
(1-x)^{-k}(1+x)^{-l} J_{n-n_{0}}^{-k,-l}(x), & n_{0}:=-(k+l) & \text { if } k, l \leq-1, \\
(1-x)^{-k} J_{n-n_{0}}^{-k, l}(x), & n_{0}:=-k & \text { if } k \leq-1, l>-1, \\
(1+x)^{-l} J_{n-n_{0}}^{k,-l}(x), & n_{0}:=-l & \text { if } k>-1, l \leq-1, \\
J_{n-n_{0}}^{k, l}(x), & n_{0}:=0 & \text { if } k, l>-1 .
\end{array}\right.
$$


The so-defined GJPs $\left\{J_{n}^{k, l}: n \geq n_{0}\right\}$ form a complete orthogonal system in $L_{\omega^{k, l}}^{2}(I)$. More importantly, as with the classical Jacobi polynomials (cf. (2.8)), they satisfy the derivative relation:

$$
\partial_{x} J_{n}^{k, l}(x)=d_{n}^{k, l} J_{n-1}^{k+1, l+1}(x),
$$

where the explicit expression of $d_{n}^{k, l}$ (behaves like $O(n)$ ) can be worked out by using Lemma 2.2 in [17]. Hence, $\left\{\partial_{x}^{r} J_{n}^{k, l}\right\}$ are mutually orthogonal with respect to the (generalized) Jacobi weight function $\omega^{k+r, l+r}$. In view of these two important properties, we can extend the analysis and the results for the classical Jacobi polynomials to the GJPs. In particular, we can extend Theorem 2.2 to the cases with both indexes being arbitrary integers.

ThEOREM 2.4. Let $\boldsymbol{\pi}_{N}^{\boldsymbol{k}, \boldsymbol{l}}$ be the $L_{\boldsymbol{\omega}^{k, l}}^{2}$-orthogonal projection upon the hyperbolic cross

$$
X_{N}^{\boldsymbol{k}, \boldsymbol{l}}:=\operatorname{span}\left\{\boldsymbol{J}_{\boldsymbol{n}}^{\boldsymbol{k}, \boldsymbol{l}}:|\boldsymbol{n}|_{\text {mix }} \leq N ; \boldsymbol{n} \geq \boldsymbol{n}_{0}\right\}, \quad \boldsymbol{k}, \boldsymbol{l} \in \mathbb{Z}^{d} .
$$

Then for any $u \in \mathcal{K}_{\boldsymbol{k}, \boldsymbol{l}}^{m}\left(I^{d}\right)$,

$$
\left\|\boldsymbol{\pi}_{N}^{\boldsymbol{k}, \boldsymbol{l}} u-u\right\|_{\mathcal{K}_{\boldsymbol{k}, \boldsymbol{l}}^{\mu}\left(I^{d}\right)} \leq D_{5} N^{\mu-m}|u|_{\mathcal{K}_{\boldsymbol{k}, l}^{m}\left(I^{d}\right)}, \quad 0 \leq \mu \leq m,
$$

where $D_{5}$ is a positive constant depending on $d, \boldsymbol{k}, \boldsymbol{l}, \mu$, and $m$, but independent of $N$.

To avoid repetition, we leave the detail of the proof to the interested reader. Notice that the explicit dependence of $D_{5}$ on $d$ can be worked out as in Theorem 2.2. In section 4 , we shall consider the application of the above result with $\boldsymbol{k}=\boldsymbol{l}=\mathbf{- 1}$.

3. Hyperbolic cross approximations in unbounded domains. In this section, we consider the hyperbolic cross approximations using Hermite and generalized Laguerre functions in unbounded domains.

3.1. Hyperbolic cross approximations by Hermite functions. We first recall some basic properties of the Hermite polynomials (cf. [28]) which are the eigenfunctions of the Sturm-Liouville problem:

$$
e^{x^{2}}\left(e^{-x^{2}} H_{n}^{\prime}(x)\right)^{\prime}+\gamma_{n} H_{n}(x)=0, \quad x \in \mathbb{R}:=(-\infty, \infty),
$$

with the corresponding eigenvalues $\gamma_{n}=2 n$. They are normalized so that

$$
\int_{-\infty}^{\infty} H_{n}(x) H_{m}(x) e^{-x^{2}} d x=\delta_{m n}
$$

The normalized Hermite polynomials satisfy

$$
H_{n}^{\prime}(x)=\sqrt{\gamma_{n}} H_{n-1}(x) \quad \Rightarrow \quad \partial_{x}^{k} H_{n}(x)=\sqrt{\mu_{n, k}} H_{n-k}(x), \quad n \geq k,
$$

where

$$
\mu_{n, k}=\prod_{j=0}^{k-1} \gamma_{n-j}=\frac{2^{k} n !}{(n-k) !}, \quad n \geq k .
$$

The univariate Hermite function is defined by

$$
\mathcal{H}_{n}(x)=e^{-x^{2} / 2} H_{n}(x), \quad n \geq 0 .
$$

We derive immediately from (3.3) and (3.5) the following recurrence relation. 
Lemma 3.1. Let $\mathcal{D}_{x}=\partial_{x}+x$. Then we have

$$
\mathcal{D}_{x}^{k} \mathcal{H}_{n}(x)=\sqrt{\mu_{n, k}} \mathcal{H}_{n-k}(x), \quad n \geq k .
$$

By (3.2), (3.5), and (3.6), $\left\{\mathcal{D}_{x}^{k} \mathcal{H}_{n}\right\}$ are mutually orthogonal in $L^{2}(\mathbb{R})$, i.e.,

$$
\int_{-\infty}^{\infty} \mathcal{D}_{x}^{k} \mathcal{H}_{n}(x) \mathcal{D}_{x}^{k} \mathcal{H}_{m}(x) d x=\mu_{n, k} \delta_{m n}
$$

As shown in [25], the use of the derivative operator $\mathcal{D}_{x}$ simplifies the analysis and leads to more precise approximation results in Sobolev spaces. Indeed, we shall see that this also facilitates the analysis in Korobov spaces.

Now, we define the multivariate Hermite functions:

$$
\mathcal{H}_{\boldsymbol{n}}(\boldsymbol{x})=\prod_{j=1}^{d} \mathcal{H}_{n_{j}}\left(x_{j}\right), \quad \boldsymbol{n} \in \mathbb{N}_{0}^{d}, \quad \boldsymbol{x} \in \mathbb{R}^{d},
$$

and the differential operator: $\mathcal{D}_{\boldsymbol{x}}^{k}:=\mathcal{D}_{x_{1}}^{k_{1}} \cdots \mathcal{D}_{x_{d}}^{k_{d}}$. It is clear that

$$
\int_{\mathbb{R}^{d}} \mathcal{D}_{x}^{k} \mathcal{H}_{n}(\boldsymbol{x}) \mathcal{D}_{\boldsymbol{x}}^{k} \mathcal{H}_{m}(\boldsymbol{x}) d \boldsymbol{x}=\boldsymbol{\mu}_{\boldsymbol{n}, k} \delta_{\boldsymbol{n} m}
$$

where

$$
\boldsymbol{\mu}_{\boldsymbol{n}, \boldsymbol{k}}=\prod_{j=1}^{d} \mu_{n_{j}, k_{j}}=\frac{2^{|\boldsymbol{k}|_{1}} n_{1} ! \cdots n_{d} !}{\left(n_{1}-k_{1}\right) ! \cdots\left(n_{d}-k_{d}\right) !}, \quad \boldsymbol{n} \geq \boldsymbol{k} .
$$

We define the Korobov-type space

$$
\mathrm{K}^{r}\left(\mathbb{R}^{d}\right)=\left\{u: \mathcal{D}_{\boldsymbol{x}}^{\boldsymbol{k}} u \in L^{2}\left(\mathbb{R}^{d}\right), 0 \leq|\boldsymbol{k}|_{\infty} \leq r\right\} \quad \forall r \in \mathbb{N}_{0}
$$

with the norm and seminorm

$$
\|u\|_{\mathcal{K}^{r}\left(\mathbb{R}^{d}\right)}=\left(\sum_{0 \leq|\boldsymbol{k}|_{\infty} \leq r}\left\|\mathcal{D}_{\boldsymbol{x}}^{\boldsymbol{k}} u\right\|_{L^{2}\left(\mathbb{R}^{d}\right)}^{2}\right)^{\frac{1}{2}}, \quad|u|_{\mathbb{K}^{r}\left(\mathbb{R}^{d}\right)}=\left(\sum_{|\boldsymbol{k}|_{\infty}=r}\left\|\mathcal{D}_{\boldsymbol{x}}^{\boldsymbol{k}} u\right\|_{L^{2}\left(\mathbb{R}^{d}\right)}^{2}\right)^{\frac{1}{2}} .
$$

As before, we define the finite-dimensional hyperbolic cross space

$$
\mathcal{S}_{N}:=\operatorname{span}\left\{\mathcal{H}_{\boldsymbol{n}}: \boldsymbol{n} \in \mathbb{N}_{0}^{d}, \quad 1 \leq|\boldsymbol{n}|_{\text {mix }} \leq N\right\},
$$

and let $P_{N} u$ be the $L^{2}$-orthogonal projection of $u$ upon $\mathcal{S}_{N}$. The notation $\Upsilon_{N, \boldsymbol{k}}^{c}$ has the same meaning as in (2.35).

TheORem 3.1. For any $u \in K^{m}\left(\mathbb{R}^{d}\right)$ and $0 \leq \boldsymbol{l} \leq m$,

$$
\left\|\mathcal{D}_{\boldsymbol{x}}^{l}\left(P_{N} u-u\right)\right\|_{L^{2}\left(\mathbb{R}^{d}\right)} \leq m^{(d-1)\left(m-|l|_{\infty}\right)}\left(\frac{m}{2}\right)^{\left(d m-|l|_{1}\right) / 2} N^{\left(|l|_{\infty}-m\right) / 2}|u|_{K^{m}\left(\mathbb{R}^{d}\right)} .
$$

Proof. Since the proof with $m=0$ is trivial, we assume $m \geq 1$. For any $u \in$ $L^{2}\left(\mathbb{R}^{d}\right)$, we derive from $(3.9)-(3.10)$ that

$$
\begin{aligned}
\left\|\mathcal{D}_{\boldsymbol{x}}^{l}\left(P_{N} u-u\right)\right\|_{L^{2}\left(\mathbb{R}^{d}\right)}^{2} & =\sum_{\boldsymbol{n} \in \Upsilon_{N, \boldsymbol{l}}^{c}} \boldsymbol{\mu}_{\boldsymbol{n}, \boldsymbol{l}}\left|\hat{u}_{\boldsymbol{n}}\right|^{2} \\
& =\sum_{\boldsymbol{n} \in \Upsilon_{N, m}^{c}} \boldsymbol{\mu}_{\boldsymbol{n}, \boldsymbol{l}}\left|\hat{u}_{\boldsymbol{n}}\right|^{2}+\sum_{\boldsymbol{n} \in \Upsilon_{N, \boldsymbol{l}}^{c} \backslash \Upsilon_{N, m}^{c}} \boldsymbol{\mu}_{\boldsymbol{n}, \boldsymbol{l}}\left|\hat{u}_{\boldsymbol{n}}\right|^{2} .
\end{aligned}
$$

Copyright (c) by SIAM. Unauthorized reproduction of this article is prohibited. 
We now deal with the first term. By (3.10),

$$
\begin{aligned}
\frac{\boldsymbol{\mu}_{\boldsymbol{n}, \boldsymbol{l}}}{\boldsymbol{\mu}_{\boldsymbol{n}, m}} & =\frac{1}{2^{m d-|l|_{1}}} \prod_{j=1}^{d} \frac{1}{\left(n_{j}-m_{j}+1\right) \cdots\left(n_{j}-l_{j}\right)} \\
& =\left(\prod_{j=1}^{d} n_{j}^{l_{j}-m}\right)\left\{\frac{1}{2^{m d-|l|_{1}}} \prod_{j=1}^{d}\left(1-\frac{m-1}{n_{j}}\right)^{-1} \cdots\left(1-\frac{l_{j}}{n_{j}}\right)^{-1}\right\} \\
& \stackrel{(2.46)}{\leq} N^{|\boldsymbol{l}|_{\infty}-m} \frac{1}{2^{m d-|l|_{1}}} \max _{\boldsymbol{n} \in \Upsilon_{N, m}^{c}}\left\{\prod_{j=1}^{d}\left(1-\frac{m-1}{n_{j}}\right)^{-1} \cdots\left(1-\frac{l_{j}}{n_{j}}\right)^{-1}\right\} \\
& :=c^{2} N^{|\boldsymbol{l}|_{\infty}-m} .
\end{aligned}
$$

Next, we derive an explicit upper bound (independent of $N$ ) of $c^{2}$. For any $\boldsymbol{n} \in \Upsilon_{N, m}^{c}$, we have $n_{j} \geq m$, and

$c^{2} \leq \frac{1}{2^{m d-|l|_{1}}} \max _{\boldsymbol{n} \in \Upsilon_{N, m}^{c}}\left\{\prod_{j=1}^{d}\left(1-\frac{m-1}{n_{j}}\right)^{l_{j}-m}\right\} \leq \frac{1}{2^{m d-|l|_{1}}} \prod_{j=1}^{d} m^{m-l_{j}} \leq\left(\frac{m}{2}\right)^{d m-|l|_{1}}$.

Hence, the first summation on the right-hand side of (3.15) has the estimate

$$
\sum_{\boldsymbol{n} \in \Upsilon_{N, m}^{c}} \boldsymbol{\mu}_{\boldsymbol{n}, \boldsymbol{l}}\left|\hat{u}_{\boldsymbol{n}}\right|^{2} \leq\left(\frac{m}{2}\right)^{d m-|l|_{1}} N^{|\boldsymbol{l}|_{\infty}-m}\left\|\mathcal{D}_{\boldsymbol{x}}^{(m, \ldots, m)} u\right\|_{L^{2}\left(\mathbb{R}^{d}\right)}^{2} .
$$

Following the proof for Case (ii) of Theorem 2.2, we are able to derive the estimate for the second summation on the right-hand side of (3.15):

$$
\sum_{\boldsymbol{n} \in \Upsilon_{N, l}^{c} \backslash \Upsilon_{N, m}^{c}} \boldsymbol{\mu}_{\boldsymbol{n}, \boldsymbol{l}}\left|\hat{u}_{\boldsymbol{n}}\right|^{2} \leq m^{2(d-1)\left(m-|l|_{\infty}\right)}\left(\frac{m}{2}\right)^{d m-|l|_{1}} N^{|l|_{\infty}-m}|u|_{\mathfrak{K}^{m}\left(\mathbb{R}^{d}\right)}^{2} .
$$

This implies the desired result.

Remark 3.1. An immediate consequence of the above theorem is

$$
\left\|P_{N} u-u\right\|_{K^{l}\left(\mathbb{R}^{d}\right)} \leq D_{6} N^{(l-m) / 2}|u|_{K^{m}\left(\mathbb{R}^{d}\right)}, \quad 0 \leq l \leq m,
$$

where $D_{6}$ is certain positive constant depending on $d, l$, and $m$.

3.2. Hyperbolic cross approximations by Laguerre functions. We start by recalling some properties of the (generalized) Laguerre polynomials, denoted by $\mathcal{L}_{n}^{(\alpha)}(x)$, which are the eigenfunctions of the Sturm-Liouville problem:

$$
x^{-\alpha} e^{x} \partial_{x}\left(x^{\alpha+1} e^{-x} \partial_{x} \mathcal{L}_{n}^{(\alpha)}(x)\right)+\lambda_{n} \mathcal{L}_{n}^{(\alpha)}(x)=0, \quad x \in \mathbb{R}_{+}:=(0, \infty),
$$

with the corresponding eigenvalues $\lambda_{n}=n$. They are mutually orthogonal and normalized so that

$$
\int_{0}^{+\infty} \mathcal{L}_{n}^{(\alpha)}(x) \mathcal{L}_{m}^{(\alpha)}(x) x^{\alpha} e^{-x} d x=\delta_{m n}, \quad \alpha>-1
$$

Copyright $@$ ㅇ by SIAM. Unauthorized reproduction of this article is prohibited. 
The normalized Laguerre polynomials satisfy the derivative relation:

$$
\partial_{x} \mathcal{L}_{n}^{(\alpha)}(x)=-\sqrt{\lambda_{n}} \mathcal{L}_{n-1}^{(\alpha+1)}(x) \quad \Rightarrow \quad \partial_{x}^{k} \mathcal{L}_{n}^{(\alpha)}(x)=(-1)^{k} \sqrt{\varrho_{n, k}} \mathcal{L}_{n-k}^{(\alpha+k)}(x),
$$

where the factor

$$
\varrho_{n, k}=n(n-1) \cdots(n-k+1), \quad n \geq k .
$$

The (generalized) Laguerre functions are

$$
L_{n}^{(\alpha)}(x)=e^{-\frac{x}{2}} \mathcal{L}_{n}^{(\alpha)}(x), \quad \alpha>-1, \quad x \in \mathbb{R}_{+} .
$$

The above relation, together with (3.23), implies the following derivative formula.

Lemma 3.2. Let $D_{x}=\partial_{x}+\frac{1}{2}$. Then we have that

$$
D_{x}^{k} L_{n}^{(\alpha)}(x)=(-1)^{k} \sqrt{\varrho_{n, k}} L_{n-k}^{(\alpha+k)}(x), \quad n \geq k .
$$

Thus, by (3.22) and (3.26),

$$
\int_{0}^{+\infty} \mathrm{D}_{x}^{k} L_{n}^{(\alpha)}(x) \mathrm{D}_{x}^{k} L_{m}^{(\alpha)}(x) x^{\alpha+k} d x=\varrho_{n, k} \delta_{m n} .
$$

Define the multivariate (generalized) Laguerre functions as

$$
\boldsymbol{L}_{\boldsymbol{n}}^{(\boldsymbol{\alpha})}(\boldsymbol{x})=\prod_{j=1}^{d} L_{n_{j}}^{\left(\alpha_{j}\right)}\left(x_{j}\right), \quad \boldsymbol{x} \in \mathbb{R}_{+}^{d}, \quad \boldsymbol{\alpha}>-1,
$$

and denote

$$
\boldsymbol{D}_{\boldsymbol{x}}^{\boldsymbol{k}}=\mathrm{D}_{x_{1}}^{k_{1}} \cdots \mathrm{D}_{x_{d}}^{k_{d}}, \quad \varpi_{\boldsymbol{\alpha}}(\boldsymbol{x})=x_{1}^{\alpha_{1}} \cdots x_{n}^{\alpha_{n}}, \quad \varrho_{\boldsymbol{n}, \boldsymbol{k}}=\prod_{j=1}^{d} \varrho_{n_{j}, k_{j}} .
$$

We have

$$
\int_{\mathbb{R}_{+}^{d}} D_{x}^{k} L_{n}^{(\boldsymbol{\alpha})}(\boldsymbol{x}) \boldsymbol{D}_{x}^{k} \boldsymbol{L}_{m}^{(\boldsymbol{\alpha})}(\boldsymbol{x}) \varpi_{\boldsymbol{\alpha}}(\boldsymbol{x}) d \boldsymbol{x}=\varrho_{n, k} \boldsymbol{\delta}_{\boldsymbol{m} n}, \quad \boldsymbol{m}, \boldsymbol{n} \geq \boldsymbol{k} .
$$

Like (3.11), we define the Korobov-type space $\boldsymbol{K}_{\boldsymbol{\alpha}}^{r}\left(\mathbb{R}_{+}^{d}\right)(r \in \mathbb{N})$, equipped with the norm and seminorm

$$
\begin{aligned}
& \|u\|_{\boldsymbol{K}_{\boldsymbol{\alpha}}^{r}\left(\mathbb{R}_{+}^{d}\right)}=\left(\sum_{0 \leq|\boldsymbol{k}|_{\infty} \leq r}\left\|\boldsymbol{D}_{\boldsymbol{x}}^{k} u\right\|_{L_{\varpi_{\alpha+\boldsymbol{k}}}^{2}\left(\mathbb{R}_{+}^{d}\right)}^{2}\right)^{\frac{1}{2}}, \\
& |u|_{\boldsymbol{K}_{\boldsymbol{\alpha}}^{r}\left(\mathbb{R}_{+}^{d}\right)}=\left(\sum_{|\boldsymbol{k}|_{\infty}=r}\left\|\boldsymbol{D}_{\boldsymbol{x}}^{k} u\right\|_{L_{\varpi_{\alpha+\boldsymbol{k}}}^{2}\left(\mathbb{R}_{+}^{d}\right)}^{2}\right)^{\frac{1}{2}} .
\end{aligned}
$$

Let $\boldsymbol{\Pi}_{N}^{(\boldsymbol{\alpha})}$ be the $L_{\varpi_{\alpha}}^{2}$-orthogonal projection upon the hyperbolic cross

$$
\boldsymbol{X}_{N}^{(\alpha)}:=\operatorname{span}\left\{\boldsymbol{L}_{\boldsymbol{n}}^{(\boldsymbol{\alpha})}: \boldsymbol{n} \in \mathbb{N}_{0}^{d},|\boldsymbol{n}|_{\text {mix }} \leq N\right\} .
$$

Following the same arguments as in the proof of the Jacobi and Hermite approximation results in Theorems 2.2 and 3.1, we can prove the following result.

Theorem 3.2. For any $u \in \boldsymbol{K}_{\boldsymbol{\alpha}}^{m}\left(\mathbb{R}_{+}^{d}\right)$,

$$
\left\|\boldsymbol{\Pi}_{N}^{(\boldsymbol{\alpha})} u-u\right\|_{\boldsymbol{K}_{\boldsymbol{\alpha}}^{l}\left(\mathbb{R}_{+}^{d}\right)} \leq D_{7} N^{(l-m) / 2}|u|_{\boldsymbol{K}_{\boldsymbol{\alpha}}^{m}\left(\mathbb{R}_{+}^{d}\right)}, \quad 0 \leq l \leq m,
$$

where $D_{7}$ is a positive constant depending on $d, l$, and $m$.

We leave the detail of the proof to the interested reader.

Copyright $@$ by SIAM. Unauthorized reproduction of this article is prohibited. 
4. An application. As an application of the hyperbolic cross approximation, we consider the sparse Legendre-Galerkin approximation to the following elliptic equation in a $d$-dimensional hypercube:

$$
-\Delta u(\boldsymbol{x})+\nu u(\boldsymbol{x})=f(\boldsymbol{x}), \quad \boldsymbol{x} \in I^{d}=(-1,1)^{d} ;\left.\quad u\right|_{\partial I^{d}}=0,
$$

where the constant $\nu \geq 0$, and $f$ is a given function.

Denote by $H_{0}^{1}\left(I^{d}\right):=\left\{u \in H^{1}\left(I^{d}\right):\left.u\right|_{\partial I^{d}}=0\right\}$. As usual, the corresponding weak formulation is to find $u \in H_{0}^{1}\left(I^{d}\right)$ such that

$$
a(\nabla u, \nabla v):=\int_{I^{d}} \nabla u \cdot \nabla v d \boldsymbol{x}+\nu \int_{I^{d}} u v d \boldsymbol{x}=\int_{I^{d}} f v d \boldsymbol{x}=(f, v)_{I^{d}} \quad \forall v \in H_{0}^{1}\left(I^{d}\right) .
$$

Let $X_{N}^{0}$ be the regular hyperbolic cross polynomial space defined by

$$
X_{N}^{0}:=\left(\sum_{1 \leq|\boldsymbol{n}|_{\text {mix }} \leq N} \bigotimes_{j=1}^{d} P_{n_{j}}(I)\right) \bigcap H_{0}^{1}\left(I^{d}\right) .
$$

The sparse Legendre-Galerkin approximation to (4.2) is to find $u_{N} \in X_{N}^{0}$ such that

$$
a\left(\nabla u_{N}, \nabla v_{N}\right)=\left(f, v_{N}\right)_{I^{d}} \quad \forall v_{N} \in X_{N}^{0} .
$$

It is clear from the definition of $a(\cdot, \cdot)$ that for $f \in L^{2}\left(I^{d}\right)$, (4.4) admits a unique solution $u_{N} \in X_{N}^{0}$. Notice that $X_{N}^{0}$ can be alternatively expressed by (cf. (2.83))

$$
X_{N}^{0}=\operatorname{span}\left\{\boldsymbol{J}_{\boldsymbol{n}}^{-1,-1}:|\boldsymbol{n}|_{\text {mix }}=\prod_{j=1}^{d} \bar{n}_{j} \leq N ; \boldsymbol{n} \geq 2\right\} .
$$

We derive the following convergence result for the scheme (4.4) by using Theorem 2.4 and a standard argument.

THEOREM 4.1. Let $u$ and $u_{N}$ be the solutions of (4.2) and (4.4), respectively. If $u \in \mathcal{K}_{-1,-1}^{m}\left(I^{d}\right) \cap H_{0}^{1}\left(I^{d}\right)$, then we have

$$
\left\|\nabla\left(u_{N}-u\right)\right\|_{L^{2}\left(I^{d}\right)} \leq D_{8} N^{1-m}|u|_{\mathcal{K}_{-1,-1}^{m}\left(I^{d}\right)}, \quad m \geq 1,
$$

where $D_{8}$ is a positive constant independent of $N$ and $u$, but depending on $d$ and $m$.

Proof. It is standard to show that

$$
\left\|\nabla\left(u_{N}-u\right)\right\|_{L^{2}\left(I^{d}\right)} \leq C\|\nabla(\phi-u)\|_{L^{2}\left(I^{d}\right)} \quad \forall \phi \in X_{N}^{0} .
$$

On the other hand, by Theorem 2.4,

$$
\begin{aligned}
& \left\|\nabla\left(\boldsymbol{\pi}_{N}^{-1,-1} u-u\right)\right\|_{L^{2}\left(I^{d}\right)} \leq \sum_{j=1}^{d}\left\|\boldsymbol{\partial}_{\boldsymbol{x}}^{\boldsymbol{e}_{j}}\left(\boldsymbol{\pi}_{N}^{-1,-1} u-u\right)\right\|_{\boldsymbol{\omega}^{\boldsymbol{e}_{j}-1, \boldsymbol{e}_{j}-1}, I^{d}} \\
& \leq C N^{1-m}|u|_{\mathcal{K}_{-1,-1}^{m}\left(I^{d}\right)},
\end{aligned}
$$

where $\boldsymbol{e}_{j}=(0, \ldots, 0,1,0, \ldots, 0)$ be the $j$ th unit vector in $\mathbb{R}^{d}$.

Thus, taking $\phi=\boldsymbol{\pi}_{N}^{-1,-1} u$ in (4.7) yields the desired result.

Copyright (C) by SIAM. Unauthorized reproduction of this article is prohibited. 
By using the GJP $J_{n}^{-1,-1}(x)$, which is proportional to the usual basis functions $L_{n-2}(x)-L_{n}(x)$ used in the Legendre-Galerkin method [24], the linear system resulting from (4.4) is sparse. However, one can no longer apply the usual technique (for the full grid) of matrix diagonalization/decomposition to efficiently solve this linear system. On the other hand, the integral on the right-hand side of (4.4) has to be approximated by a suitable quadrature or the function $f$ has to be replaced by a suitable interpolation $I_{N} f$. All of these implementation details as well as how to efficiently solve the resultant linear systems will be the subject of a forthcoming investigation [26].

5. Concluding remarks. We considered in this paper hyperbolic cross approximations using Jacobi polynomials for bounded domains and Hermite and generalized Laguerre functions for unbounded domains, and we established optimal error estimates in proper anisotropic weighted Korobov spaces for both regular hyperbolic cross approximations and optimized hyperbolic cross approximations. These results were proved systematically with a uniform approach that can be used to study the hyperbolic cross approximations by other orthogonal systems.

It was shown that for functions with regularity in the proper anisotropic weighted Korobov spaces, the convergence rates of regular hyperbolic cross approximations (in the proper anisotropic weighted Korobov spaces) deteriorate only very mildly with respect to the dimension $d$ (cf. Theorems 2.2, 3.1, and 3.2). Moreover, the convergence rates of the optimized hyperbolic cross approximations are independent of the dimension $d$ (cf. Theorem 2.3). These results are analogous to the results for hyperbolic cross approximations based on Fourier series (cf. [15]), except that the proper functional spaces are the anisotropic weighted Korobov spaces, instead of the spaces with bounded mixed derivatives for periodic functions.

While we have only considered an application of the approximation results to a spectral Galerkin method for the Poisson-type equation in a hypercube, it is clear that these hyperbolic cross approximation results are of general interest and can be used for a large class of high-dimensional problems.

\section{REFERENCES}

[1] R. A. Adams, Sobolov Spaces, Academic Press, New York, 1975.

[2] A. Bandrauk, M. C. Delfour, and C. Le Bris, eds., High-Dimensional Partial Differential Equations in Science and Engineering, CRM Proc. Lecture Notes. 41, AMS, Providence, RI, 2007.

[3] V. Barthelmann, E. Novak, And K. Ritter, High dimensional polynomial interpolation on sparse grids, Adv. Comput. Math., 12 (2000), pp. 273-288.

[4] G. Baszenski and F.-J. Delvos, A discrete Fourier transform scheme for Boolean sums of trigonometric operators, in Multivariate Approximation Theory, IV (Oberwolfach, 1989), Internat. Ser. Numer. Math. 90, Birkhäuser, Basel, 1989, pp. 15-24.

[5] R. Bellman, Adaptive Control Processes: A Guided Tour, Princeton University Press, Princeton, NJ, 1961.

[6] C. Bernardi and Y. Maday, Spectral method, in Handbook of Numerical Analysis, Vol. 5 (Part 2), P. G. Ciarlet and L. L. Lions, eds., North-Holland, Amsterdam, 1997, pp. 209485.

[7] H.-J. Bungartz and M. Griebel, Sparse grids, Acta Numer., 13 (2004), pp. 147-269.

[8] C. Canuto, M. Y. Hussaini, A. Quarteroni, and T. A. Zang, Spectral Methods: Fundamentals in Single Domains, Sci. Comput., Springer-Verlag, Berlin, 2006.

[9] R. Courant and D. Hilbert, Methods of Mathematical Physics, Vol. 1, Interscience Publishers, New York, 1953.

[10] M. Dauge And R. Stevenson, Sparse Tensor Product Wavelet Approximation of Singular Functions, preprint, 2009. 
[11] N. M. Dobrovol'skil And A. L. Roshchenya, On the number of points in a lattice in a hyperbolic cross, Mat. Zametki, 63 (1998), pp. 363-369.

[12] I. P. Gavrilyuk, W. Hackbusch, and B. N. KhoromskiJ, Hierarchical tensor-product approximation to the inverse and related operators for high-dimensional elliptic problems, Computing, 74 (2005), pp. 131-157.

[13] V. Gradinaru, Fourier transform on sparse grids: Code design and the time dependent Schrödinger equation, Computing, 80 (2007), pp. 1-22.

[14] M. GRIEBEL, Sparse grids and related approximation schemes for higher dimensional problems, in Foundations of Computational Mathematics (Santander, 2005), London Math. Soc. Lecture Note Ser. 331, Cambridge University Press, Cambridge, UK, 2006, pp. 106-161.

[15] M. Griebel and J. Hamaekers, Sparse grids for the Schrödinger equation, M2AN Math. Model. Numer. Anal., 41 (2007), pp. 215-247.

[16] B.-Y. Guo, J. Shen, AND L.-L. WANG, Optimal spectral-Galerkin methods using generalized Jacobi polynomials, J. Sci. Comput., 27 (2006), pp. 305-322.

[17] B.-Y. Guo, J. Shen, And L.-L. WANG, Generalized Jacobi polynomials/functions and their applications, Appl. Numer. Math., 59 (2009), pp. 1011-1028.

[18] B.-Y. Guo AND L.-L. Wang, Jacobi approximations in non-uniformly Jacobi-weighted Sobolev spaces, J. Approx. Theory, 128 (2004), pp. 1-41.

[19] K. HallatscheK, Fouriertransformation auf dünnen Gittern mit hierarchischen Basen, Numer. Math., 63 (1992), pp. 83-97.

[20] N. M. Korobov, On the approximate solution of integral equations, Dokl. Akad. Nauk SSSR, 128 (1959), pp. 233-238 (in Russian).

[21] N. M. Korobov, Exponential Sums and Their Applications, Math. Appl. (Soviet Ser.) 80, Kluwer Academic, Dordrecht, The Netherlands, 1992; translated from the 1989 Russian original by Yu. N. Shakhov.

[22] C. Schwab and R. Stevenson, Adaptive wavelet algorithms for elliptic PDE's on product domains, Math. Comp., 77 (2008), pp. 71-92.

[23] C. Schwab, E. Süli, AND R. A. Todor, Sparse finite element approximation of highdimensional transport-dominated diffusion problems, M2AN Math. Model. Numer. Anal., 42 (2008), pp. 777-819.

[24] J. Shen, Efficient spectral-Galerkin method I. Direct solvers of second-and fourth-order equations using Legendre polynomials, SIAM J. Sci. Comput., 15 (1994), pp. 1489-1505.

[25] J. Shen And L.-L. WAng, Some recent advances on spectral methods for unbounded domains, Commun. Comput. Phys., 5 (2009), pp. 195-241.

[26] J. SHEn AND H. YU, Efficient spectral sparse grid methods for high dimensional elliptic problems, SIAM J. Sci. Comput., submitted.

[27] F. Sprengel, Interpolation of functions from Besov-type spaces on Gauß-Chebyshev grids, J. Complexity, 16 (2000), pp. 507-523.

[28] G. Szegö, Orthogonal Polynomials, 4th ed., AMS Coll. Publ. 23, AMS, Providence, RI, 1975.

[29] V. Temlyakov, Approximation of Functions with Bounded Mixed Derivative, Proc. Steklov Inst. Math. 178, AMS, Providence, RI, 1989.

[30] C. Zenger, Sparse grids, in Parallel Algorithms for Partial Differential Equations, W. Hackbusch, ed., Notes Numer. Fluid Mech. 31, Vieweg, Braunschweig, 1991, pp. 297-301.

Copyright $@$ by SIAM. Unauthorized reproduction of this article is prohibited. 